\title{
COARSE SPACES FOR FETI-DP AND BDDC METHODS FOR HETEROGENEOUS PROBLEMS: CONNECTIONS OF DEFLATION AND A GENERALIZED TRANSFORMATION-OF-BASIS APPROACH*
}

\author{
AXEL KLAWONN ${ }^{\dagger \ddagger}$, MARTIN KÜHN ${ }^{\S}$, AND OLIVER RHEINBACH ${ }^{\| \uparrow}$
}

\begin{abstract}
In FETI-DP (Finite Element Tearing and Interconnecting) and BDDC (Balancing Domain Decomposition by Constraints) domain decomposition methods, the convergence behavior of the iterative scheme can be improved by implementing a coarse space using a transformation of basis and local assembly. This is an alternative to coarse spaces implemented by deflation or balancing. The transformation-of-basis approaches are more robust with respect to inexact solvers than deflation and therefore more suitable for multilevel extensions. In this paper, we show a correspondence of FETI-DP or BDDC methods using a generalized transformation-of-basis approach and of FETI-DP methods using deflation or balancing, where the deflation vectors are obtained from the transformation of basis. These methods then have essentially the same eigenvalues. As opposed to existing theory, this result also applies to general scalings and highly heterogeneous problems.

We note that the new methods differ slightly from the classic FETI-DP and BDDC methods using a transformation of basis and that the classic theory has to be replaced. An important application for the theory presented in this paper are FETI-DP and BDDC methods with adaptive coarse spaces, i.e., where deflation vectors are obtained from approximating local eigenvectors. These methods have recently gained considerable interest.
\end{abstract}

Key words. domain decomposition, FETI-DP, BDDC, coarse space, deflation, transformation of basis, change of variables, elliptic partial differential equations

\section{AMS subject classifications. 65N30,65N25,65N50,65N55,74E05}

1. Introduction. In this section, we first present the state-of-the-art of domain decomposition methods including adaptively computed coarse spaces with a focus on FETI-DP (Finite Element Tearing and Interconnecting-Dual Primal) and BDDC (Balancing Domain Decomposition by Constraints) methods. We then give a brief overview of the main result which is about the relation of the spectra of FETI-DP and BDDC methods using a generalized transformation-of-basis approach and a related deflation or balancing methods to implement the new, e.g., adaptively computed coarse space components. The theoretical and numerical results of this paper have already been made public in April 2017 in a technical report [34]; the present manuscript is a completely revised and reorganized version. Also note that the results presented in our recently published article [33] are applications of the theory presented here.

1.1. State-of-the-art. The numerical solution of partial differential equations by finite elements often requires a fine discretization of the given domain in order to obtain a good approximate solution of the original problem. This leads to large sparse linear systems of equations, which often cannot be solved by sparse direct solvers. Instead, iterative methods are used. Domain decomposition methods [62] are widely-used iterative algorithms for the parallel solution of implicit finite element problems. In these methods, the finite element

*Received April 27, 2018. Accepted October 16, 2019. Published online on January 29, 2020. Recommended by $\mathrm{O}$. Widlund. This manuscript is part of a PhD. Thesis project of the second author in Applied Mathematics at Universität zu Köln (Mathematische-Naturwissenschaftliche Fakultät, Köln, Germany); see [46]. The thesis was successfully defended on April 20, 2018.

$\dagger$ University of Cologne, Department of Mathematics and Computer Science, Weyertal 86-90, 50931 Köln, Germany, https://www. numerik. uni-koeln. de (axel.klawonn@uni-koeln.de).

${ }^{\ddagger}$ University of Cologne, Center for Data and Simulation Science, https://www. cds. uni-koeln. de.

${ }^{\S}$ CERFACS (Centre Européen de Recherche et de Formation Avancée en Calcul Scientifique), 42 Avenue Gaspard Coriolis, 31057 Toulouse Cedex 01, France (martin. kuehn@ cerfacs. fr).

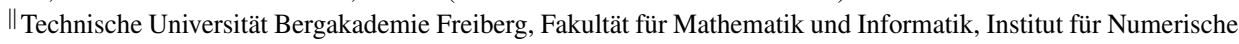
Mathematik und Optimierung, 09596 Freiberg, Germany; (oliver.rheinbach@math.tu-freiberg. de).

๑ Technische Universität Bergakademie Freiberg, Universitätsrechenzentrum (URZ), 09596 Freiberg, Germany. 


\section{ETNA}

Kent State University and

Johann Radon Institute (RICAM)

problem is decomposed into independent, parallel local problems. Additionally, to obtain scalability in the number of subdomains, a coarse space has to ensure global transport of information.

Originally, the coarse spaces of FETI-DP and BDDC domain decomposition methods were formed by coupling the subdomains in a few primal variables, i.e., by a partial finite element assembly used to enforce continuity across subdomain boundaries at the vertices of subdomains [62]. In three dimensions, this is not sufficient to obtain a good condition number bound. A remedy is to make edge averages continuous across the interface in each iteration. A transformation of basis, to explicitly introduce the averages as new variables followed by partial finite element assembly in these new variables, is a well-known technique of implementing such average constraints in FETI-DP and BDDC methods; see, e.g., [39, 43, 44, 47, 58]. An alternative is the use of the edge characteristic functions as deflation vectors in a deflation or balancing approach; see, e.g., [27, 41] and the references therein. However, the transformationof-basis approach is more reliable than deflation or balancing, being more robust with respect to inexact solvers; see, e.g., [41]. It therefore allows us to replace the direct coarse solver by a preconditioner as, e.g., in $[36,57]$ for better scalability. Other techniques to enforce the primal constraints are given in [19], where additional global Lagrange multipliers are used, or in $[10,48,49]$, where local saddle point problems are used. We will also briefly comment on some of these approaches in the following paragraphs; a detailed analysis of all approaches is, however, outside the scope of this paper, which primarily considers the relation between the deflation and transformation-of-basis approaches for FETI-DP. We will also show how to transfer the new theory of the generalized transformation-of-basis approach for FETI-DP to BDDC.

For highly heterogeneous problems, e.g., in almost incompressible elasticity with coefficient jumps, domain decomposition methods can be equipped with automatic or adaptive coarse spaces if the method otherwise does not converge or if the convergence is very slow. To the best of our knowledge such adaptive coarse spaces were first introduced to domain decomposition in [3, 4]. More recently, these approaches have attracted substantial interest, and many new methods with provable bounds have been proposed. In all of these methods, instead of using coarse spaces defined (and analyzed) a priori, the adaptive (i.e., problem-specific) coarse spaces are constructed automatically during the computation; see, e.g., [49, 21, 22, 52, 50, 14, 12, 61, 60, 37, 38, 30, 32, 23, 26, 1, 54, 7, 63, 31, 55, 17, 5, 64, 33], given in historical order.

For almost a decade, the first approach mentioned in the list above, [49], lacked a condition number bound for the adaptive FETI-DP operator. In [38], a condition number bound for the coarse space of [49] was provided for two dimensions. In [32], we were then able to provide such a bound for FETI-DP in three dimensions after an additional enrichment using a small number of edge eigenvalue problems. However, in [32], we used FETI-DP with deflation or balancing which limits the potential for parallel scalability because the coarse problem then has to be solved exactly.

In [49], local saddle point problems are used in an alternative coarse space implementation. Such saddle point problems were also used in the classic BDDC method using a priori constraints [10]; later they were used for adaptive BDDC [49]. We note that the first condition number bound for the adaptive algorithm in [49], which was given in [38], makes use of deflation. The same applies for the theory in [32]. The results of this paper are therefore also of interest for the adaptive approach given in [49].

In this paper, for a given FETI-DP method using balancing or deflation (under a few assumptions, see Section 5), we construct a FETI-DP method with a (generalized) transformationof-basis approach. We then show that in both approaches, the eigenvalues of the preconditioned 
operator are the same, except possibly for eigenvalues at 0 and 1 ; see Theorem 6.7. The relation to a BDDC method using a transformation of basis is given in Theorem 7.3. As a result, the approach [32] can now also be formulated using a (generalized) transformation-of-basis approach for FETI-DP and BDDC; the resulting method has been described and tested in [33], which include preliminary parallel results.

Several papers in the literature implicitly rely on the existence of a (generalized) transformation-of-basis approach as described in this paper, corresponding to the FETI-DP method using deflation. This includes our own paper [33] as well as [1, 7, 31, 54, 55], where adaptive FETI-DP or BDDC methods are combined with a change of variables and partial finite element assembly.

It has already been shown in [27, 41], but only for homogeneous problems (and multiplicity scaling), that for any FETI-DP and BDDC method with a transformation of basis there exists a corresponding FETI-DP method using deflation (also known as projector preconditioning) or balancing with essentially the same spectrum; see [41, Theorem 6.7]. Note that the reverse is not always true without additional assumptions, notably the FETI-DP method using deflation in [24] for almost incompressible elasticity (which uses sums of face averages as constraints) cannot be implemented using the generalized transformation-of-basis approach.

In the present paper, the correspondence relation from [27, 41] is generalized to heterogeneous problems with jumps along and across subdomain boundaries. An important difference to $[27,41]$ comes from the scaling since for heterogeneous problems, in addition to the automatic coarse spaces, a suitable scaling might be indispensable. Otherwise the adaptive coarse space can become large; see, e.g., [33, 38].

We revisit the standard theory and, under certain assumptions on the constraints, we show a correspondence between a FETI-DP method using deflation and a FETI-DP or BDDC method using a generalized transformation-of-basis approach. The arguments of the standard theory are replaced by our Lemmas 6.3 and 6.4 .

For readers who are familiar with the standard theory of FETI-DP and BDDC methods, let us note that we discuss how the transformed $P_{D}$ operator, which is central to the theory of FETI-DP and BDDC, is defined; see [62, p. 175], [44, p. 172], or [42, p. 1551] for the standard definition of $P_{D}$. Note that it is denoted $P_{\Delta}$ in $[44,62]$. We then show in Theorem 6.7 that the corresponding methods using a transformation of basis, deflation, or balancing have essentially the same spectrum. We define a new, transformed scaling (see Definition 7.1) which in some cases will be identical to the initial scaling (cf. [41]), e.g., for multiplicity scaling or for scalings which are constant on edges or faces, while not true in general. Even if the original scaling is diagonal (as is the case with $\rho$-scaling), the transformed scaling is in general not diagonal. For FETI-DP, we can also provide a transformation of basis in the space of Lagrange multipliers; see Section 5.3. This transformation is performed implicitly in the algorithm.

The generalized transformation-of-basis approach introduced in this paper slightly differs from the classical approach; the theory and also the implementation are different. The reason is that in deflation, using nondiagonal scaling (e.g., deluxe) can yield an interaction between deflated and non-deflated variables; see Figure 1.1. Such an interaction is not present in the standard transformation-of-basis approach as in [44, 47, 43, 39]; see Figure 1.2. This is relevant even for diagonal scalings (other than multiplicity), since a transformation of basis for heterogeneous problems will in general result in a nondiagonal scaling; see Definition 7.1. Using the standard transformation-of-basis approach, these interactions are lost. We briefly comment on the alternative implementation of coarse constraints in BDDC using saddle point problems as in [10]. In the saddle point approach, the interactions are lost if the primal variables are held nonredundantly and apart from the remaining dual variables. If the primal 


\section{ETNA}

Kent State University and

Johann Radon Institute (RICAM)

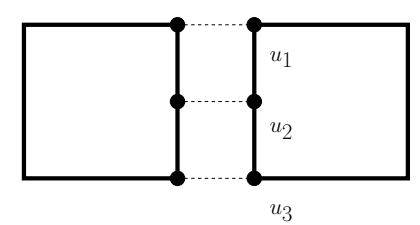

FIG. 1.1. Let $\bar{u}=u_{1}+u_{2}+u_{3}$ be the edge average corresponding to a constraint enforced by deflation. Then, even for a diagonal scaling $D$, in $B^{T} D B$ there can be an interaction between the average $\bar{u}$ and the other degrees of freedom. To preserve this interaction, in the generalized transformation-of-basis approach we keep the Lagrange multipliers for a posteriori primal variables despite of the partial assembly; see Section 5.2. The dotted lines symbolize the three Lagrange multipliers.

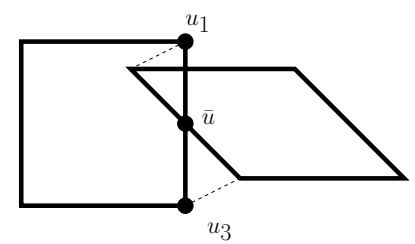

FIG. 1.2. Using the standard transformation-of-basis approach results in fewer Lagrange multipliers compared to Figure 1.1. An interaction of the primal edge average $\bar{u}$ with the other degrees of freedom through the scaling $D$ is not present; $c f$. (5.5) and (5.6). The dotted lines symbolize the two Lagrange multipliers.

variables are held redundantly, as are all other interface variables, and are scaled correctly, then the interactions could possibly be implemented correctly in a way similar to the generalized transformation-of-basis approach. Note, however, that several papers on adaptive FETI-DP and BDDC methods in their theory routinely make use of the equivalence to a transformationof-basis approach [30, 32, 63, 1, 54, 7, 31, 55]. The generalized transformation-of-basis approach, however, preserves the necessary information. A simple example to illustrate the loss of information in the standard approach is given in Section 4.2.

The focus of the present paper is thus on the construction, for heterogeneous problems, of a generalized transformation-of-basis approach for FETI-DP and BDDC with full theoretical justification and to show the equivalence to a corresponding FETI-DP method with a deflation or balancing approach. It is natural to combine our approaches presented here with adaptive coarse spaces, e.g., as in our approach in [32] or for the many other adaptive coarse space approaches developed recently. This, however, is outside the scope of this paper and is considered in [33].

The use of the generalized transformation-of-basis approach enables the implementation of a competitive parallel FETI-DP improving the standard FETI-DP approach for chosen heterogeneous problems, both with respect to time and precision; see [33, 46, 35].

The remainder of the paper is structured as follows. In the following section, we try to spell out the main result of this paper by mentioning the important identities derived in this article but without going into details of the notation. In Section 2, we will introduce our elliptic model problem and introduce the underlying finite element geometry used in the domain decomposition approach. In Section 3, we present the standard FETI-DP and BDDC algorithm with a transformation of basis and deflation or balancing. In Section 4, we will give a short motivation for the need of our generalized transformation-of-basis approach. In Section 5, we introduce a generalized variant of the transformation-of-basis approach for arbitrary scalings, coefficient distributions, and constraint vectors. In Section 6, we will finally prove that FETI-DP with the generalized transformation-of-basis approach has the same 
nontrivial spectrum as FETI-DP with a deflation or balancing approach. The corresponding theory and a short discussion on how to apply the generalized transformation-of-basis approach for BDDC follows in Section 7. In Section 8, we will present some results to visualize our theoretical findings. Finally, we draw some conclusions in Section 9.

1.2. Main result. In this section, we briefly describe our main result without dwelling on the details of notation for which we refer to the later sections of this article. For quite general problems, including heterogeneous elliptic finite element problems and general scalings, we show the equality of the spectra of the deflated preconditioned FETI-DP operator $(I-P) M_{D}^{-1}(I-P)^{T} F$ and that of a new preconditioned FETI-DP operator $\widehat{M}_{D}^{-1} \widehat{F}$ using a new basis constructed by a transformation of basis from the deflation vectors, i.e.,

$$
\sigma\left((I-P) M_{D}^{-1}(I-P)^{T} F\right)=\sigma\left(\widehat{M}_{D}^{-1} \widehat{F}\right),
$$

where $\sigma$ denotes the spectrum. Note that the index $D$ in $M_{D}^{-1}$ as well as $\widehat{M}_{D}^{-1}$ refers to the standard FETI-DP Dirichlet preconditioner [62] and not to deflation.

The new algorithm, denoted the generalized transformation-of-basis approach, is different from known methods since in the scaling on the subdomain interface, an interaction of primal and dual variables can occur. This is possible since we keep Lagrange multipliers corresponding to non-nodal primal variables in the FETI-DP system.

As a result, the standard theory does not apply anymore and has to be replaced; cf. equation (1.1).

We also show that a corresponding, transformed BDDC preconditioned system also essentially has the same eigenvalues, i.e., that

$$
\sigma\left(\widehat{M}_{\mathrm{BDDC}}^{-1} \mathcal{S}\right) \backslash\{0,1\} \subset \sigma\left(\widehat{M}_{D}^{-1} \widehat{F}\right)=\sigma\left(M_{P P}^{-1} F\right) .
$$

Our results thus generalize the findings from [41] to general heterogeneous problems.

The new theory is also of interest for recent adaptive FETI-DP and BDDC methods [1, 7, $12,30,31,32,37,38,49,50,54,55,63]$. Some adaptive FETI-DP approaches are formulated using deflation. For these approaches, our results allow us to define FETI-DP and BDDC methods without deflation but with the same convergence properties. Other adaptive FETIDP or BDDC approaches use a transformation of basis combined with partial assembly but implicitly make use of the results presented in this paper.

In contrast to deflation or balancing approaches, the transformation-of-basis approaches allow for a more flexible treatment of the coarse problem in domain decomposition including the use of inexact solvers, e.g., $[11,36,57]$, which is crucial to obtain scalability to the order of million cores [36] and beyond.

2. Model problem and geometry. Given a bounded polyhedral domain $\Omega \subset \mathbb{R}^{3}$ where the Dirichlet boundary $\partial \Omega_{D} \subset \partial \Omega$ is a closed subset of positive surface measure and $\partial \Omega_{N}:=\partial \Omega \backslash \partial \Omega_{D}$, we consider the weak formulation of an elliptic problem

$$
a(u, v)=F(v) \quad \forall v \in H_{0}^{1}\left(\Omega, \partial \Omega_{D}\right) .
$$

The theory presented here is purely algebraic and therefore, in particular, applies to the diffusion problem and linear elasticity. In this paper, we present numerical results for the diffusion equation. There, we have

$$
a(u, v):=\int_{\Omega} \rho \nabla u \cdot \nabla v \mathrm{~d} x \quad \text { and } \quad F(v):=\int_{\Omega} f v \mathrm{~d} x+\int_{\partial \Omega_{N}} g v \mathrm{~d} s .
$$


For numerical results on linear elasticity and adaptive FETI-DP or BDDC, see [33, 46].

The domain $\Omega$ is decomposed into $N$ nonoverlapping open subdomains $\Omega_{i}, i=1, \ldots, N$. The interface $\Gamma$ is defined as the union of the interior subdomain boundaries, i.e., $\Gamma:=\left\{x \in \bar{\Omega}_{i} \cap \bar{\Omega}_{j} ; i \neq j\right\}$. The subdomains are then triangulated and then discretized by the finite element method with matching nodes on the interface. For simplicity, we use piecewise linear conforming finite elements.

In three dimensions, the interface can be decomposed into vertices, edges, and faces; for a detailed definition of these sets, cf. [43]. Edges and faces are defined as open sets. A face shared by two subdomains $\Omega_{i}$ and $\Omega_{j}$ will be denoted by $\mathcal{F}^{i j}$ while we denote edges shared by $\Omega_{i}, \Omega_{j}, \Omega_{k}$ and possibly more subdomains by $\mathcal{E}^{i k}$. Vertices of $\Omega_{i}$ that belong to multiple subdomains are denoted by $\mathcal{V}^{i l}$.

By $W^{h}\left(\Omega_{i}\right), i=1, \ldots, N$, we denote the local finite element space on $\Omega_{i}$. The local trace space $W_{i}:=W^{h}\left(\Gamma_{i}\right)$ is defined on $\Gamma_{i}:=\overline{\Omega_{i}} \cap \Gamma$. We also introduce the global product space $W:=\Pi_{i=1}^{N} W_{i}$ and denote the space of functions that are continuous across the interface by $\widehat{W} \subset W$.

3. FETI-DP and BDDC with a transformation of basis and FETI-DP with deflation and balancing. To make this paper self-contained, in this section we recall standard FETI-DP and BDDC methods with a transformation of basis and FETI-DP with a deflation or balancing approach. Note that the deflation or balancing approach for BDDC is different from that for the BDDC method with a transformation of basis and is therefore not described; see [41].

Transformation of basis, deflation, or balancing provide ways of adding additional constraints to the coarse space of the underlying FETI-DP or BDDC domain decomposition method. Another possibility is the use of optional Lagrange multipliers; see [19, 25, 43, 48, 49]. For more details on FETI-DP and BDDC methods; see, e.g., [9, 10, 18, 19, 20, 62] and, e.g., $[39,43,44,47]$ for the use of a transformation of basis in FETI-DP and BDDC as well as, e.g., $[15,16,27,41,51,53]$ for FETI-DP with deflation or balancing.

\subsection{Standard FETI-DP and BDDC methods.}

3.1.1. The standard FETI-DP algorithm. We partition the set of degrees of freedom into interior, dual, and primal degrees of freedom, denoted by an index $I, \Delta^{\prime}$, and $\Pi^{\prime}$, respectively. Interior degrees of freedom are associated with nodes in the interior of subdomains and on the Neumann boundary $\partial \Omega_{N}$. Dual and primal degrees of freedom belong to nodes on the interface $\Gamma$, and every node on $\Gamma$ can be classified as one of them. In contrast to many other works on standard FETI-DP and BDDC, we use the indices $\Delta^{\prime}$ and $\Pi^{\prime}$ instead of $\Delta$ and $\Pi$ since the latter will be reserved for the generalized transformation-of-basis approach; cf. Sections 4-7.

For every subdomain $\Omega_{i}, i=1, \ldots, N$, we assemble the local stiffness matrix $K^{(i)}$ and the local load vector $f^{(i)}$. The vectors $u$ are partitioned into those of the interior degrees of freedom $u_{I}$, the dual degrees of freedom $u_{\Delta^{\prime}}$, and the primal degrees of freedom $u_{\Pi^{\prime}}$. Assuming an appropriate ordering of the degrees of freedom, we obtain the following partitioning of the local stiffness matrices and the solution and load vectors

$$
K^{(i)}=\left[\begin{array}{ccc}
K_{I I}^{(i)} & K_{\Delta^{\prime} I}^{(i) T} & K_{\Pi^{\prime} I}^{(i) T} \\
K_{\Delta^{\prime} I}^{(i)} & K_{\Delta^{\prime} \Delta^{\prime}}^{(i)} & K_{\Pi^{\prime} \Delta^{\prime}}^{(i) T} \\
K_{\Pi^{\prime} I}^{(i)} & K_{\Pi^{\prime} \Delta^{\prime}}^{(i)} & K_{\Pi^{\prime} \Pi^{\prime}}^{(i)}
\end{array}\right], \quad u^{(i)}=\left[\begin{array}{c}
u_{I}^{(i)} \\
u_{\Delta^{\prime}}^{(i)} \\
u_{\Pi^{\prime}}^{(i)}
\end{array}\right], \quad \text { and } f^{(i)}=\left[\begin{array}{c}
f_{I}^{(i)} \\
f_{\Delta^{\prime}}^{(i)} \\
f_{\Pi^{\prime}}^{(i)}
\end{array}\right] .
$$

Further, we introduce the following matrices and vectors

$$
K_{B B}^{(i)}:=\left[\begin{array}{cc}
K_{I I}^{(i)} & K_{\Delta^{\prime} I}^{(i) T} \\
K_{\Delta^{\prime} I}^{(i)} & K_{\Delta^{\prime} \Delta^{\prime}}^{(i)}
\end{array}\right], \quad K_{\Pi^{\prime} B}^{(i)}:=\left[K_{\Pi^{\prime} I}^{(i)} K_{\Pi^{\prime} \Delta^{\prime}}^{(i)}\right], \quad \text { and } f_{B}^{(i)}:=\left[f_{I}^{(i) T} f_{\Delta^{\prime}}^{(i) T}\right]^{T}
$$


as well as

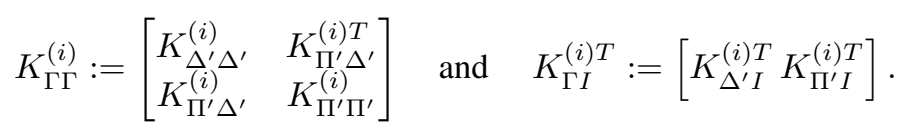

Next, we define the global block matrices

$$
K_{I I}:=\operatorname{diag}_{i=1}^{N} K_{I I}^{(i)}, \quad K_{\Delta^{\prime} \Delta^{\prime}}:=\operatorname{diag}_{i=1}^{N} K_{\Delta^{\prime} \Delta^{\prime}}^{(i)}, \quad \text { and } \quad K_{\Pi^{\prime} \Pi^{\prime}}:=\operatorname{diag}_{i=1}^{N} K_{\Pi^{\prime} \Pi^{\prime}}^{(i)}
$$

as well as

$$
K_{B B}:=\operatorname{diag}_{i=1}^{N} K_{B B}^{(i)}, \quad K_{\Gamma \Gamma}:=\operatorname{diag}_{i=1}^{N} K_{\Gamma \Gamma}^{(i)},
$$

and where $K_{\Gamma I}$ is the corresponding global off-diagonal block.

We also need assembly operators. The first one, $R_{\Pi^{\prime}}^{T}:=\left[R_{\Pi^{\prime}}^{(1) T}, \ldots, R_{\Pi^{\prime}}^{(N) T}\right]$, performs the assembly in the primal variables $u_{\Pi^{\prime}}^{(i)}$ and is needed for both FETI-DP and BDDC. The second one, $R_{\Delta^{\prime}}^{T}:=\left[R_{\Delta^{\prime}}^{(1) T}, \ldots, R_{\Delta^{\prime}}^{(N) T}\right]$, performs assembly in the dual variables $u_{\Delta^{\prime}}^{(i)}$ and is only needed for BDDC. The transposed operators $R_{\Pi^{\prime}}$ and $R_{\Delta^{\prime}}$ are then extension operators that distribute global information to the local subdomains.

For FETI-DP, in place of $R_{\Delta^{\prime}}$, we need a jump operator $B_{\Gamma}=\left[B_{\Gamma}^{(1)}, \ldots, B_{\Gamma}^{(N)}\right]$ which is built from values 0 and \pm 1 such that $B_{\Gamma} u=0$ holds for $u \in \widehat{W}$.

By an assembly in the primal variables, we obtain

$$
\begin{aligned}
\widetilde{K}_{\Pi^{\prime} \Pi^{\prime}} & =\sum_{i=1}^{N} R_{\Pi^{\prime}}^{(i) T} K_{\Pi^{\prime} \Pi^{\prime}}^{(i)} R_{\Pi^{\prime}}^{(i)}, & \widetilde{K}_{\Pi^{\prime} B} & =\left[R_{\Pi^{\prime}}^{(1) T} K_{\Pi^{\prime} B}^{(1)}, \ldots, R_{\Pi^{\prime}}^{(N) T} K_{\Pi^{\prime} B}^{(N)}\right], \\
\widetilde{f} & =\left[f_{B}^{T},\left(\sum_{i=1}^{N} R_{\Pi^{\prime}}^{(i) T} f_{\Pi^{\prime}}^{(i)}\right)^{T}\right]^{T}, & \text { and } & \widetilde{S}_{\Pi^{\prime} \Pi^{\prime}}=\widetilde{K}_{\Pi^{\prime} \Pi^{\prime}}-\widetilde{K}_{\Pi^{\prime} B} K_{B B}^{-1} \widetilde{K}_{\Pi^{\prime} B}^{T} .
\end{aligned}
$$

Then, the FETI-DP master system is given by

$$
\left[\begin{array}{ccc}
K_{B B} & \widetilde{K}_{\Pi^{\prime} B}^{T} & B_{B}^{T} \\
\widetilde{K}_{\Pi^{\prime} B} & \widetilde{K}_{\Pi^{\prime} \Pi^{\prime}} & 0 \\
B_{B} & 0 & 0
\end{array}\right]\left[\begin{array}{c}
u_{B} \\
\widetilde{u}_{\Pi^{\prime}} \\
\lambda
\end{array}\right]=\left[\begin{array}{c}
\widetilde{f} \\
0
\end{array}\right] .
$$

Here, $B_{B}$ is the nontrivial part of $B$ and has the form $B_{B}=\left[B_{B}^{(1)}, \ldots, B_{B}^{(N)}\right]$. The (unpreconditioned) FETI-DP system

$$
F \lambda=d
$$

is obtained after elimination of $\widetilde{u}^{T}=\left[u_{B}^{T}, \widetilde{u}_{\Pi^{\prime}}^{T}\right]^{T}$, where

$$
\begin{aligned}
F & =\left[\begin{array}{ll}
B_{B} & 0
\end{array}\right]\left[\begin{array}{cc}
K_{B B} & \widetilde{K}_{\Pi^{\prime} B}^{T} \\
\widetilde{K}_{\Pi^{\prime} B} & \widetilde{K}_{\Pi^{\prime} \Pi^{\prime}}
\end{array}\right]^{-1}\left[\begin{array}{c}
B_{B}^{T} \\
0^{T}
\end{array}\right] \\
& =B_{B} K_{B B}^{-1} B_{B}^{T}+B_{B} K_{B B}^{-1} \widetilde{K}_{\Pi^{\prime} B}^{T} \widetilde{S}_{\Pi^{\prime} \Pi^{\prime}}^{-1} \widetilde{K}_{\Pi^{\prime} B} K_{B B}^{-1} B_{B}^{T}=B_{\Gamma} \widetilde{S}^{-1} B_{\Gamma}^{T} \\
d & =B_{B} K_{B B}^{-1} f_{B}+B_{B} K_{B B}^{-1} \widetilde{K}_{\Pi^{\prime} B}^{T} \widetilde{S}_{\Pi^{\prime} \Pi^{\prime}}^{-1}\left(\left(\sum_{i=1}^{N} R_{\Pi^{\prime}}^{(i) T} f_{\Pi^{\prime}}^{(i)}\right)-\widetilde{K}_{\Pi^{\prime} B} K_{B B}^{-1} f_{B}\right) .
\end{aligned}
$$


Here, the Schur complement $\widetilde{S}_{\Pi^{\prime} \Pi^{\prime}}$ is defined as $\widetilde{S}_{\Pi^{\prime} \Pi^{\prime}}:=\widetilde{K}_{\Pi^{\prime} \Pi^{\prime}}-\widetilde{K}_{\Pi^{\prime} B^{\prime}} K_{B^{\prime} B^{\prime}}^{-1} \widetilde{K}_{\Pi^{\prime} B^{\prime}}^{T}$.

For heterogeneous problems, the scaling is an important ingredient of FETI-DP and BDDC methods. We will introduce two kind of commonly used scalings. We first introduce the standard $\rho$-scaling; see, e.g., [40, 42, 56, 59, 62]. For $x \in \Gamma_{i}$ we introduce $\mathcal{N}_{x}$ as the set of indices of subdomains that have $x$ on their boundaries. We define the coefficient evaluation by $\widehat{\rho}_{i}(x):=\sup _{x \in \operatorname{supp}\left(\varphi_{x}\right) \cap \Omega_{i}} \rho(x)$. Here, $\varphi_{x}$ is the nodal finite element function at $x$ and $\operatorname{supp}\left(\varphi_{x}\right)$ its support. Let $\Omega_{j}$ and $\Omega_{i}$ share either a face or an edge, and let $x \in \partial \Omega_{i} \cap \partial \Omega_{j}$. The corresponding nontrivial row of $B^{(j)}$ is then multiplied by the scaling $\delta_{i}^{\dagger}(x):=\widehat{\rho}_{i}(x) / \sum_{k \in \mathcal{N}_{x}} \widehat{\rho}_{k}(x)$, and we obtain the local scaling $D^{(j)}$. In BDDC the degrees of freedom on $\partial \Omega_{i}$ are scaled by $\delta_{i}^{\dagger}(x)$ defining the scaling $D_{u}^{(i)}$; see (3.3).

Secondly, we introduce deluxe scaling; see, e.g, $[2,6,8,12,13]$. Then, the Schur complement of the stiffness matrix on the interface $\Gamma$, i.e.,

$$
S_{\Gamma \Gamma}:=\operatorname{diag}_{i=1}^{N} S^{(i)}:=\operatorname{diag}_{i=1}^{N}\left(K_{\Gamma \Gamma}^{(i)}-K_{\Gamma I}^{(i)}\left(K_{I I}^{(i)}\right)^{-1} K_{\Gamma I}^{(i) T}\right)
$$

has to be defined. Let us consider either a face $\mathcal{F}^{i j}$ shared by the two subdomains $\Omega_{i}$ and $\Omega_{j}$ or an edge $\mathcal{E}^{i k}$ shared by the subdomains $\Omega_{i}, \Omega_{j}, \Omega_{k}$. Multiplicities greater than three can be handled analogously.

For $l \in\{i, j\}$, the matrix $S^{(l)}$ is partitioned as

$$
S^{(l)}=\left[\begin{array}{cc}
S_{\mathcal{F}^{i j} \mathcal{F}^{i j}}^{(l)} & S_{\mathcal{F}^{i j} \mathcal{F}^{i j} C}^{(l)} \\
S_{\mathcal{F}^{i j} \mathcal{F}^{i j}}^{(l)} & S_{\mathcal{F}^{i j} \mathcal{F}^{i j} C}^{(l)}
\end{array}\right] \quad \text { and } \quad S^{(l)}=\left[\begin{array}{cc}
S_{\mathcal{E}^{i k} \mathcal{E}^{i k}}^{(l)} & S_{\mathcal{E}^{i k} \mathcal{E}^{i k} C}^{(l)} \\
S_{\mathcal{E}^{i k} \mathcal{E}^{i k}}^{(l)} & S_{\mathcal{E}^{i k} \mathcal{E}^{i k} C}^{(l)}
\end{array}\right] ;
$$

see, e.g., [38]. Then, for $l \in\{i, j\}$ we define $S_{\mathcal{F}^{i j}, 0}^{(l)}:=S_{\mathcal{F}^{i j} \mathcal{F}^{i j}}^{(l)}$ and $S_{\mathcal{E}^{i k}, 0}^{(l)}:=S_{\mathcal{E}^{i k} \mathcal{E}^{i k}}^{(l)}$.

In deluxe scaling the nontrivial rows of $B^{(j)}$ corresponding to the Lagrange multipliers of a face $\mathcal{F}^{i j}$ are multiplied by $D_{u, \mathcal{F}^{i j}}^{(i)}=\left(S_{\mathcal{F}^{i j}, 0}^{(i)}+S_{\mathcal{F}^{i j}, 0}^{(j)}\right)^{-1} S_{\mathcal{F}^{i j}, 0}^{(i)}$ if the orientation of the constraints in $B$ are chosen consistently. Otherwise, some entries of $D_{u, \mathcal{F}^{i j}}^{(i)}$ have to be multiplied by -1 .

For an edge $\mathcal{E}^{i k}$, in deluxe scaling the nontrivial rows of the matrix $B_{\Gamma}^{(j)}$ corresponding to the Lagrange multipliers coupling $\Omega_{i}$ and $\Omega_{j}$ on this edge are multiplied by $D_{u, \mathcal{E}^{i k}}^{(i)}=\left(S_{\mathcal{E}^{i k}, 0}^{(i)}+S_{\mathcal{E}^{i k}, 0}^{(j)}+S_{\mathcal{E}^{i k}, 0}^{(k)}\right)^{-1} S_{\mathcal{E}^{i k}, 0}^{(i)}$. Again, a consistent orientation of the Lagrange multipliers is assumed. The rows of $B_{\Gamma}^{(j)}$ corresponding to Lagrange multipliers coupling $\Omega_{j}$ and $\Omega_{k}$ on $\mathcal{E}^{i k}$ and the rows of $B_{\Gamma}^{(i)}$ and $B_{\Gamma}^{(k)}$ are scaled analogously. The final scaling matrix $D^{(j)}$ is obtained from the local scaling matrices on the faces and edges of $\Omega_{j}$. In BDDC, the degrees of freedom on $\mathcal{F}^{i j}$ and $\mathcal{E}^{i k}$ of $\partial \Omega_{i}$ are scaled by $D_{u, \mathcal{F}^{i j}}^{(i)}$ and $D_{u, \mathcal{E}^{i k}}^{(i)}$, respectively, defining the scaling $D_{u}^{(i)}$ for the BDDC preconditioner; see (3.3). Let us note that for the case of $\rho$-scaling, we have $D^{(i) T}=D^{(i)}$, for $i=1, \ldots, N$, while the deluxe scaling matrix is not symmetric.

The standard preconditioner for the FETI-DP system is known as the Dirichlet preconditioner; see [62]. We introduce the restriction operator $R_{\Gamma}$, which restricts a vector $\tilde{u}$ to the interface by removing values at the interior nodes and its transpose $R_{\Gamma}^{T}$, which extends a vector $u_{\Gamma}$ by zero.

For both kinds of scalings, we obtain the scaled jump operator

$$
B_{D, \Gamma}=\left[B_{D, \Gamma}^{(1)}, \ldots, B_{D, \Gamma}^{(N)}\right]=\left[D^{(1) T} B_{\Gamma}^{(1)}, \ldots, D^{(N) T} B_{\Gamma}^{(N)}\right] .
$$

The standard Dirichlet preconditioner can then be written (see [62])

$$
M_{D}^{-1}:=B_{D, \Gamma} R_{\Gamma}^{T} S_{\Gamma \Gamma} R_{\Gamma} B_{D, \Gamma}^{T}=B_{D, \Gamma} \widetilde{S} B_{D, \Gamma}^{T} .
$$


3.1.2. Standard FETI-DP theory. In standard FETI-DP theory, condition number bounds are constructed by estimating the Rayleigh quotient

$$
\frac{\left\langle M_{D}^{-1} F \lambda, \lambda\right\rangle_{F}}{\langle\lambda, \lambda\rangle_{F}}
$$

see, e.g., [43, Theorem 8.2]. By $\widetilde{W}$, we denote the finite element functions from $W$ that are assembled in the a priori primal variables. We have $\widehat{W} \subset \widetilde{W} \subset W$; see the end of Section 2 .

For arbitrary $\lambda$ and $u:=\widetilde{S}^{-1} B^{T} \lambda \in \widetilde{W}$, we have

$$
\left\langle M_{D}^{-1} F \lambda, \lambda\right\rangle_{F}=\left\langle B_{D} \widetilde{S} B_{D}^{T} B \widetilde{S}^{-1} B^{T} \lambda, B \widetilde{S}^{-1} B^{T} \lambda\right\rangle=\left\langle P_{D} u, P_{D} u\right\rangle_{\widetilde{S}}=\left|P_{D} u\right|_{\widetilde{S}}^{2} .
$$

If we can prove that

$$
\left|P_{D} u\right|_{\widetilde{S}} \leq C|u|_{\widetilde{S}}
$$

with a constant $C>0$, then we obtain the upper bound $\left\langle M_{D}^{-1} F \lambda, \lambda\right\rangle_{F} \leq C\langle\lambda, \lambda\rangle_{F}$; see [43, the proof of the upper bound in Theorem 8.2]. From a Raleigh quotient argument, we then have an upper bound for the maximum eigenvalue $\lambda_{\max }\left(M_{D}^{-1} F\right) \leq C$. For the minimum eigenvalue of $M_{D}^{-1} F$ it can be shown that 1 is a lower bound; see [43, Theorem 8.2]. The lower bound can be obtained by simple linear algebra and by using that $B P_{D} u=B u$ for $u \in \widetilde{W}$; for the latter equality, see [43, formula (8.1)]. Then, for the lower bound, we obtain

$$
\begin{aligned}
\langle\lambda, \lambda\rangle_{F} & =\left\langle B \widetilde{S}^{-1} B^{T} B_{D} \widetilde{S}^{1 / 2} \widetilde{S}^{-1 / 2} B^{T} \lambda, \lambda\right\rangle=\left\langle\widetilde{S}^{-1 / 2} B^{T} \lambda, \widetilde{S}^{1 / 2} B_{D}^{T} B \widetilde{S}^{-1} B^{T} \lambda\right\rangle \\
& \leq\left\langle\widetilde{S}^{-1 / 2} B^{T} \lambda, \widetilde{S}^{-1 / 2} B^{T} \lambda\right\rangle^{1 / 2}\left\langle\widetilde{S}^{1 / 2} B_{D}^{T} B \widetilde{S}^{-1} B^{T} \lambda, \widetilde{S}^{1 / 2} B_{D}^{T} B \widetilde{S}^{-1} B^{T} \lambda\right\rangle^{1 / 2} \\
& =\langle\lambda, \lambda\rangle_{F}{ }^{1 / 2}\left\langle M_{D}^{-1} F \lambda, \lambda\right\rangle^{1 / 2} ;
\end{aligned}
$$

see [57, Theorem 2.4.2] for the proof provided here.

Hence, from (3.2), we obtain the condition number estimate

$$
\kappa\left(M_{D}^{-1} F\right) \leq C,
$$

where $C$ is the positive constant from (3.2) and $\kappa$ denotes the spectral condition number.

3.1.3. The standard BDDC algorithm. For BDDC, we will use a different ordering $\left[u_{\Delta^{\prime}}^{(1)}, \ldots, u_{\Delta^{\prime}}^{(N)}, u_{\Pi^{\prime}}^{(1)}, \ldots, u_{\Pi^{\prime}}^{(N)}\right]$ instead of $\left[u_{\Delta^{\prime}}^{(1)}, u_{\Pi^{\prime}}^{(1)}, \ldots, u_{\Delta^{\prime}}^{(N)}, u_{\Pi^{\prime}}^{(N)}\right]$. We first need $\mathcal{K}_{\Gamma \Gamma}$ and $\mathcal{K}_{\Gamma I}$ to define the Schur complement on the interface $\Gamma$,

$$
\mathcal{S}_{\Gamma \Gamma}:=\mathcal{K}_{\Gamma \Gamma}-\mathcal{K}_{\Gamma I} K_{I I}^{-1} \mathcal{K}_{\Gamma I}^{T} .
$$

The right-hand side is then given by $\left[g_{\Delta^{\prime}}^{T}, g_{\Pi^{\prime}}^{T}\right]^{T}$, which is obtained by eliminating the interior degrees of freedom from $f$. The BDDC system matrix is the fully assembled global Schur complement, which can also be written as

$$
\mathcal{S}:=\left[\begin{array}{cc}
R_{\Delta^{\prime}}^{T} & 0 \\
0 & I_{\Pi^{\prime}}
\end{array}\right]\left[\begin{array}{cc}
I_{\Delta^{\prime}} & 0 \\
0 & R_{\Pi^{\prime}}^{T}
\end{array}\right] \mathcal{S}_{\Gamma \Gamma}\left[\begin{array}{cc}
I_{\Delta^{\prime}} & 0 \\
0 & R_{\Pi^{\prime}}
\end{array}\right]\left[\begin{array}{cc}
R_{\Delta^{\prime}} & 0 \\
0 & I_{\Pi^{\prime}}
\end{array}\right] .
$$

The BDDC system for $u_{\Gamma}^{T}=\left[u_{\Delta^{\prime}}^{T}, u_{\Pi^{\prime}}^{T}\right]^{T}$ with the corresponding right-hand side $g^{T}=\left[g_{\Delta^{\prime}}^{T} R_{\Delta^{\prime}}, g_{\Pi^{\prime}}^{T} R_{\Pi^{\prime}}\right]^{T}$ is given by

$$
\mathcal{S} u_{\Gamma}=g
$$


Using the scaling $D_{u}$ introduced in Section 3.1.1, we can define the scaled assembly operator $R_{\Delta^{\prime}, D_{u}}^{T}$ acting on the dual degrees of freedom $\Delta^{\prime}$. The standard BDDC preconditioner then reads in our notation as

$$
M_{\mathrm{BDDC}}^{-1}:=\left[\begin{array}{cc}
R_{\Delta^{\prime}, D_{u}}^{T} R_{\Gamma} & 0 \\
0 & I_{\Pi^{\prime}}
\end{array}\right]\left[\begin{array}{cc}
K_{B B} & \widetilde{K}_{\Pi B}^{T} \\
\widetilde{K}_{\Pi B} & \widetilde{K}_{\Pi \Pi}^{T}
\end{array}\right]^{-1}\left[\begin{array}{cc}
R_{\Gamma}^{T} R_{\Delta^{\prime}, D_{u}} & 0 \\
0 & I_{\Pi^{\prime}}
\end{array}\right] .
$$

3.2. Transformation of basis in FETI-DP and BDDC methods. Let us now briefly recall the standard transformation-of-basis approach for FETI-DP and BDDC with a constant scaling for any face and edge, e.g., multiplicity scaling or $\rho$-scaling for certain coefficient distributions; see, e.g., [39, 41, 43, 44, 47]. Using partial finite element assembly, continuity across the subdomain boundary of certain degrees of freedom can be enforced for the finite element function. Using a transformation of basis from a nodal to a different basis, general constraints can be enforced using the same technique.

Consider an edge $\mathcal{E}$ shared by $\Omega_{i}, \Omega_{j}$ and other subdomains. Suppose that during the Krylov iteration the iterates $u$ should fulfill a constraint given by the normalized vector $c$ defined on $\partial \Omega_{i} \cap \mathcal{E}$ (and equal to that on $\partial \Omega_{j} \cap \mathcal{E}$ ), i.e., such that

$$
c^{T}\left(u_{\mathcal{E}}^{(i)}-u_{\mathcal{E}}^{(j)}\right)=0 \quad \Leftrightarrow \quad c^{T} u_{\mathcal{E}}^{(i)}=c^{T} u_{\mathcal{E}}^{(j)}
$$

for $u_{\mathcal{E}}^{(l)}=u_{\mid \partial \Omega_{l} \cap \mathcal{E}}, l \in\{i, j\}$. For instance, $c=\frac{1}{n_{\mathcal{E}}}(1, \ldots, 1)^{T}$ represents a common edge average shared by $\Omega_{i}$ and $\Omega_{j}$, where $n_{\mathcal{E}}$ is the length of $c$, an example of the use of a non-nodal basis function.

We refer to equations such as (3.4) as constraint and $c$ as a constraint vector. We then define a (square) transformation matrix

$$
T_{\mathcal{E}}^{(l)}=\left[c, C^{(l) \perp}\right], \quad l \in\{i, j\},
$$

where $C^{(l) \perp}$ is chosen such that $T_{\mathcal{E}}^{(l)}$ is orthogonal. We then define the transformation matrix $T^{(l)}$, which acts on the complete subdomain boundary and which is identical to $T_{\mathcal{E}}^{(l)}$ on the edge $\mathcal{E}$ and the identity elsewhere. We obtain the transformed variables $\bar{u}^{(l)}$, the stiffness matrices $\bar{K}^{(l)}$, and the load vectors $\bar{f}^{(l)}$ on $\Omega_{l}$ as

$$
\bar{K}^{(l)}=T^{(l) T} K^{(l)} T^{(l)}, \quad \bar{u}^{(l)}=T^{(l) T} u^{(l)}, \quad \bar{f}^{(l)}=T^{(l) T} f^{(l)}, \quad l \in\{i, j\} .
$$

After this transformation of basis has been performed, an assembly in the new (a posteriori) primal variables is used to enforce the given constraint.

This procedure indeed enforces our original constraint corresponding to $c$ as follows. We have

$$
c^{T} u_{\mathcal{E}}^{(l)}=c^{T}\left[c, C^{(l) \perp}\right] \bar{u}_{\mathcal{E}}^{(l)}=\bar{u}_{\mathcal{E}, 1}^{(l)}, \quad l \in\{i, j\},
$$

where $\bar{u}_{\mathcal{E}, 1}^{(l)}$ is the value at the first degree of freedom of the edge $\mathcal{E}$. Let us now identify the variables $\bar{u}_{\mathcal{E}, 1}^{(i)}$ and $\bar{u}_{\mathcal{E}, 1}^{(j)}$ by using partial finite element assembly of this degree of freedom. We denote the new values on this edge by $\widehat{u}_{\mathcal{E}}^{(l)}$ with $\widehat{u}_{\mathcal{E}, 1}:=\widehat{u}_{\mathcal{E}, 1}^{(i)}:=\widehat{u}_{\mathcal{E}, 1}^{(j)}:=\frac{1}{2} \bar{u}_{\mathcal{E}, 1}^{(i)}+\frac{1}{2} \bar{u}_{\mathcal{E}, 1}^{(j)}$ for the first degree of freedom and $\widehat{u}_{\mathcal{E}, k}^{(l)}=\bar{u}_{\mathcal{E}, k}^{(l)}$ for $k>1$. For the values transformed back to the initial basis, we now find that

$$
c^{T} T_{\mathcal{E}}^{(i)} \widehat{u}_{\mathcal{E}}^{(i)}=\frac{1}{2} \bar{u}_{\mathcal{E}, 1}^{(i)}+\frac{1}{2} \bar{u}_{\mathcal{E}, 1}^{(j)}=c^{T} T_{\mathcal{E}}^{(j)} \widehat{u}_{\mathcal{E}}^{(j)},
$$

i.e., the constraint is enforced. 
3.3. Deflation and balancing in FETI-DP methods. In this section, we briefly explain the deflation and the balancing approach for FETI-DP. Our presentation is based on [41, Section 2 and Section 5] and [32, Section 4]. Note that the deflation or balancing approach for BDDC is different from the BDDC method with a transformation of basis, and therefore it will not be described; see [41].

Deflation (see [53]) is also known as projector preconditioning; see [15]. Deflation (Projector Preconditioning) and Balancing approaches are used in order to add additional constraints to the FETI-DP coarse space. Short introductions to deflation and balancing in the context of FETI-DP and domain decomposition methods can be found in [15, 16, 27, 41, 51, 53] and the references therein.

In the following, for a matrix $A$ we will denote by $A^{+}$an arbitrary pseudoinverse of $A$ satisfying $A A^{+} A=A$ and $A^{+} A A^{+}=A^{+}$. The following description is an extension of the presentation in [41] to the case of a semidefinite matrix $F$; it can be found in detail in [32]. Let $U=\left(u_{1}, \ldots, u_{s}\right)$ be given as the matrix where the constraint vectors $u_{i}, i=1, \ldots, s$, for the Lagrange multipliers are stored as columns. We then define

$$
P:=U\left(U^{T} F U\right)^{+} U^{T} F
$$

and multiply the FETI-DP system by $(I-P)^{T}$. This yields the deflated system

$$
(I-P)^{T} F \lambda=(I-P)^{T} d
$$

which is still consistent. Since range $(U) \subset \operatorname{ker}\left((I-P)^{T} F\right)$, we have that range $(F(I-P)) \subset \operatorname{ker}\left(U^{T}\right)$ also for a semidefinite matrix $F$. Since $(I-P)^{T}$ is also a projection, we have

$$
(I-P)^{T} F=F(I-P)=(I-P)^{T} F(I-P),
$$

and therefore only the components of the dual variable in range $(I-P)$ are relevant to the construction of the Krylov spaces. Let $\lambda^{*}$ denote the solution of the original system $F \lambda=d$, which is unique only up to an element in $\operatorname{ker} B^{T}$. Let $\hat{\lambda} \in \operatorname{range}(I-P)$ be a solution of (3.6). Then, $\hat{\lambda}$ is identical to $(I-P) \lambda^{*}$ up to an element in $\operatorname{ker} B^{T}$. We have the decomposition

$$
\lambda^{*}=P \lambda^{*}+(I-P) \lambda^{*}=: \bar{\lambda}+(I-P) \lambda^{*},
$$

where $\bar{\lambda}$ can be expressed as $\bar{\lambda}=P \lambda^{*}=U\left(U^{T} F U\right)^{+} U^{T} F F^{+} F \lambda^{*}=P F^{+} d$. As already argued in [32], the solution in terms of $u$ does not change if $(I-P) \lambda^{*}$ is replaced by $\hat{\lambda}$, i.e.,

$$
u_{\Delta}=\widetilde{S}^{-1}\left(\widetilde{f}_{\Delta}-B^{T} \lambda^{*}\right)=\widetilde{S}^{-1}\left(\widetilde{f}_{\Delta}-B^{T}(\bar{\lambda}+\hat{\lambda})\right) .
$$

Preconditioning the deflated system of equations by the Dirichlet preconditioner $M_{D}^{-1}$ defined in (3.1) gives

$$
M_{D}^{-1}(I-P)^{T} F \lambda=M_{D}^{-1}(I-P)^{T} d .
$$

Another multiplication with $(I-P)$ from the left gives the (symmetric) deflation or projector preconditioner

$$
M_{P P}^{-1}:=(I-P) M_{D}^{-1}(I-P)^{T} .
$$

As shown in [41, Theorem 6.1], the nonzero eigenvalues are not changed. The deflated and preconditioned problem can then be written: Find $\lambda \in \operatorname{range}(I-P)$ such that

$$
M_{P P}^{-1} F \lambda=M_{P P}^{-1} d .
$$




\section{ETNA}

Kent State University and

Johann Radon Institute (RICAM)

Instead of computing $\bar{\lambda}$ a posteriori, the computation can be executed iteratively in the solver. This gives the balancing preconditioner $M_{B P}^{-1}:=M_{P P}^{-1}+P F^{+}$. As already stated in [32], the balancing preconditioner for a semidefinite matrix $F$ is of the form $M_{B P}^{-1}=M_{P P}^{-1}+U\left(U^{T} F U\right)^{+} U^{T} F F^{+}$, but we can equivalently use

$$
M_{B P}^{-1}=M_{P P}^{-1}+U\left(U^{T} F U\right)^{+} U^{T}
$$

since it will be applied to $F \lambda=d$. Using [41] and [32], we find that the eigenvalues of $M_{B P}^{-1} F$ and $M_{P P}^{-1} F$ are essentially the same.

4. Motivation for a generalized transformation-of-basis approach for general scalings and arbitrary constraints.

4.1. Context and nomenclature. When FETI-DP and BDDC methods are combined with deflation or balancing, typically an initial coarse space is defined, which introduces a sufficient coupling to obtain invertibility of the subdomain problems. A simple vertex coarse space can suffice as an initial coarse space. At the same time, an initial scaling is chosen; see, e.g., Section 5.2. For heterogeneous problems, the scaling used in the preconditioner (i.e., $\rho$-scaling, deluxe scaling, etc.) is important to obtain a robust iterative method. Then, a second coarse space is implemented by deflation or balancing to obtain faster convergence; see, e.g., [27, 41]. In this paper, we will denote the first coarse space also as the "a priori coarse space" and the second coarse space, defined after the scaling, as "a posteriori coarse space".

In adaptive FETI-DP and BDDC methods [1, 7, 12, 30, 31, 32, 33, 37, 38, 49, 50, 54, $55,64]$, the a posteriori coarse space is highly dependent on the a priori scaling since the computation of the approximate eigenvectors for the a posteriori coarse space makes use of the a priori scaling. Indeed, the choice of an inappropriate a priori scaling (e.g., use of multiplicity scaling for heterogeneous problems) will lead to an (unnecessarily) large a posteriori coarse space. This can be observed, e.g., in [38]. We will denote the index set $\Pi^{\prime}$ introduced in Section 3.1 as the "a priori set of primal variables". After a transformation of the standard basis, we use an additional partial finite element assembly in the index set $\Pi$ (our a posteriori coarse space). Thus, the final set of primal variables is $\Pi^{\prime} \cup \Pi$. The index set of the final (or remaining) dual variables is therefore $\Delta=\Delta^{\prime} \backslash \Pi$. We denote the index set $\Pi$ as the "a posteriori set of primal variables".

Let us briefly illustrate the difference between the a priori and a posteriori coarse spaces by considering a corresponding deflation method. Let us assume that after an initial coarse space and a scaling $D$ have been defined, based on the use of this scaling, a deflation vector $c_{D}:=c(D)$ has been chosen to further accelerate convergence. As an example, $c_{D}$ could be defined by the solution of local eigenvalue problems as they appear in adaptive domain decomposition methods (see $[1,7,12,30,31,32,33,37,38,49,50,54,55,64])$ and where the a priori defined scaling appears in the eigenvalue problems explicitly or implicitly. The deflated method using the constraint $c_{D}^{T} B w=0$ may allow the construction of a bound

$$
\left|P_{D} w\right|_{\widetilde{S}}^{2} \leq C|w|_{\widetilde{S}}^{2} \quad \forall w \in\left\{w \in \widetilde{W}: c_{D}^{T} B w=0\right\} ;
$$

cf. Section 3. However, the estimate (4.1) depends on the use of the scaling $D$ in combination with the constraint $c_{D}^{T} B w=0$ and may not be valid anymore if a different scaling $\widetilde{D}$ is used.

It is important to note that after the transformation, a diagonal scaling may not be diagonal anymore. This occurs for non-nodal degrees of freedom such as edge averages. For nodal degrees of freedom, an interaction between dual and primal variables also results when a nondiagonal scaling such as deluxe scaling is used. The interaction between dual and primal variables is not present in the classical theory, and a standard argument used in the classical 

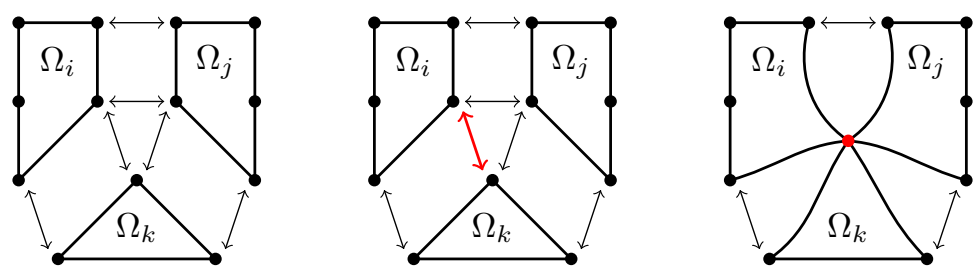

FIG. 4.1. Cross sectional view of three subdomains $\Omega_{i}, \Omega_{j}$, and $\Omega_{k}$ sharing the edge $\mathcal{E}^{i k}$. Arrows symbolize redundant Lagrange multipliers in FETI-DP (left). Assume that, using deflation, one primal constraint is introduced involving the Lagrange multiplier depicted in bold red color (center). In our implementation of the (generalized) transformation-of-basis, continuity is now enforced between all three nodes using partial assembly (right).

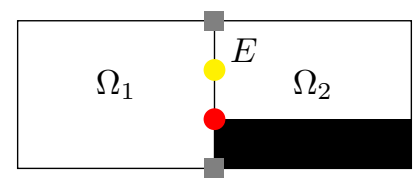

FIG. 4.2. Decomposition of $\Omega=[0,1] \times\left[0, \frac{1}{2}\right]$ into two subdomains $\Omega_{1}, \Omega_{2}$ with homogeneous Dirichlet boundary conditions on $\partial \Omega$ and given coefficient distribution. A non-homogeneous coefficient distribution with $\rho_{1}=1$ (white) and $\rho_{2}=1$ e6 (black) is considered. Initial primal variables $\left(\Pi^{\prime}\right)$ are indicated by gray squares. Initial dual variables are indicated by circles. The red circle represents an a posteriori primal variable $\left(\Pi^{\prime}\right)$, i.e., here, we enforce a scaling-dependent constraint by a transformation of basis. The yellow circle represents the remaining dual variable.

theory, i.e., that iterates are zero in the primal variables, cannot be used anymore. In our theory, the use of Lemma 6.3 and Lemma 6.4 replaces this standard argument. Constructing the scaling for the transformed variables only in the remaining dual variables will in general not give the desired result.

Then, for adaptive methods for instance, we can reduce a corresponding estimate for the transformed $\widehat{P}_{D}$ operator to an already existing estimate as in [32].

This discussion is relevant for FETI-DP and BDDC methods with adaptive coarse spaces where first a scaling is chosen (e.g., $\rho$ - or deluxe-scaling) and then a coarse space is constructed based on this scaling. We therefore believe that this is also of interest for the analysis of other adaptive approaches $[1,7,31,54,55]$. The details of adaptive FETI-DP and BDDC methods in combination with the generalized transformation-of-basis are discussed in [33].

Let us now revisit the classical theory considering an example where the assumption of diagonal and constant scaling on any face and any edge, as assumed in [41], is not fulfilled.

4.2. Example. We now show that the generalized transformation-of-basis approach can lead to nonzero values in non-nodal primal variables even for a diagonal scaling.

Consider the edge $E$ with nodes $\Pi$ (red circle) and $\Delta$ (yellow circle) between the two subdomains $\Omega_{1}$ and $\Omega_{2}$ as depicted in Figure 4.2.

The $\rho$-scaling for the degrees of freedom for the nodal basis is given by

$$
D_{u}^{(1)}=\operatorname{diag}\left(\frac{1}{1+1 \mathrm{e} 6}, \frac{1}{2}\right) \quad \text { and } \quad D_{u}^{(2)}=I-D_{u}^{(1)} .
$$

Then, assume a (nondiagonal) transformation $T$ of the form

$$
T=\left[\begin{array}{ll}
t_{r r} & t_{r y} \\
t_{y r} & t_{y y}
\end{array}\right]
$$

with $t_{y r}, t_{r y} \neq 0, T^{T} T=I$, and where the first column is given by a scaling dependent constraint vector $c_{D}^{T}=\left[t_{r r}, t_{y r}\right]^{T}$ providing an estimate such as (4.1). The constraint is 
scaling-dependent since the estimate can only be obtained when the constraint is used in combination with the given scaling. This is, e.g., typically the case in methods with adaptive coarse spaces. If the scaling in the adaptive methods is changed, e.g., by restriction (see below), then the condition number bound of the adaptive method may not be valid anymore.

The indices $y$ and $r$ in (4.2) refer to the nodes colored yellow (y) and red (r) in Figure 4.2. In the new basis, the transformed $\rho$-scaling (see Definition 7.1) is

$$
\begin{aligned}
& \widehat{D}_{u}^{(1)}=\left[\begin{array}{cc}
\frac{1}{1+1 \mathrm{e} 6} t_{r r}^{2}+\frac{1}{2} t_{y r}^{2} & \frac{1}{1+1 \mathrm{e} 6} t_{r r} t_{r y}+\frac{1}{2} t_{y r} t_{y y} \\
\frac{1}{1+1 \mathrm{ee} 6} t_{r r} t_{r y}+\frac{1}{2} t_{y r} t_{y y} & \frac{1}{1+1 \mathrm{e} 6} t_{r y}^{2}+\frac{1}{2} t_{y y}^{2}
\end{array}\right]=:\left[\begin{array}{ll}
\alpha & \beta \\
\gamma & \delta
\end{array}\right] \quad \text { and } \\
& \widehat{D}_{u}^{(2)}=I-\widehat{D}_{u}^{(1)}=\left[\begin{array}{cc}
1-\alpha & -\beta \\
-\gamma & 1-\delta
\end{array}\right]
\end{aligned}
$$

After enforcing continuity in the a posteriori primal variable, we have with $w_{\Pi}=w_{1, \Pi}=$ $w_{2, \Pi,}$

$$
\begin{aligned}
\left(\widehat{P}_{D} w\right)_{\Pi}^{(1)}=\left(\left(I-\widehat{E}_{D_{u}}\right) w\right)_{\Pi}^{(1)} & =w_{\Pi}-\left(\alpha w_{\Pi}+\beta w_{1, \Delta}+(1-\alpha) w_{\Pi}-\beta w_{2, \Delta}\right) \\
& =-\beta\left(w_{1, \Delta}-w_{2, \Delta}\right) .
\end{aligned}
$$

Since in general $\beta \neq 0$ and $w_{1, \Delta} \neq w_{2, \Delta}$, we obtain in general a nonzero value in the a posteriori primal variables after $\widehat{P}_{D}=I-\widehat{E}_{D_{u}}$ is applied; this is contrary to the assumptions of the standard theory. The interaction of a posteriori primal and dual variables was also observed in [31].

Neither the use of the standard scaling $\left(D_{u}^{(1)}\right)_{\Delta}=\frac{1}{2}$ nor the transformed and restricted scaling $\left(\widehat{D}_{u}^{(1)}\right)_{\Delta}=\frac{1}{1+1 \mathrm{e} 6} t_{r y}^{2}+\frac{1}{2} t_{y y}^{2}$ are adequate here.

5. Correspondence of FETI-DP using a generalized transformation-of-basis approach to FETI-DP using deflation or balancing.

5.1. Preliminaries and solution spaces. In this section, we consider the FETI-DP method in the transformed variables. For convenience, we order the primal variables first.

We show that for every FETI-DP or BDDC method with a generalized transformation-ofbasis approach, there exists a corresponding FETI-DP method using deflation or balancing with essentially the same eigenvalues. The reverse is true under certain conditions; see Assumption 1.

As in the standard transformation-of-basis approach, the constraints are stored in the columns of the transformation matrix $T$. The transformation $T$ transforms from a basis, e.g., with explicit averages or adaptive constraints, to the standard nodal basis. The transformation $T$ is block diagonal with respect to the subdomains. We assume that the columns of $T$ are orthonormal, and we can use $T^{T}$, the transpose of $T$, to compute its inverse $T^{-1}$. Note that $T^{T}$ has to be replaced by $T^{-1}$ if $T$ is not orthogonal. The transformed variables are denoted by $\bar{w}$ or locally by $\bar{w}^{(i)}, i=1, \ldots, N$.

In the following, we make the following assumption for each edge and each face. For simplicity, consider a single edge $\mathcal{Z}_{1}=\mathcal{Z}_{l_{1}}, l \in\{i, j, k\}$, common to the three subdomains $\Omega_{i}, \Omega_{j}$, and $\Omega_{k}$. Without loss of generality, we can assume that the transformations $T_{\mathcal{Z}_{i_{1}}}^{(i)}, T_{\mathcal{Z}_{j_{1}}}^{(j)}, T_{\mathcal{Z}_{k_{1}}}^{(k)}$ on the subdomain edges $\mathcal{Z}_{1} \cap \Omega_{i}, \mathcal{Z}_{1} \cap \Omega_{j}$, and $\mathcal{Z}_{1} \cap \Omega_{k}$ are identical. This implies that the numbering of the edge nodes is consistent for all three subdomains.

Here, we also implicitly use the assumption that a constraint vector does not span several faces and/or edges, which is not valid, e.g., for the small FETI-DP coarse space in [24] introduced for almost incompressible elasticity; see Assumption 1. 
We then need an assembly operator $R^{T}$ in the a posteriori primal variables. In contrast to the standard method, we also need the multiplicity-weighted assembly operator for the a posteriori primal variables defined by

$$
R_{\mu}^{T}:=\left(R^{T} R\right)^{-1} R^{T},
$$

and we therefore have $R_{\mu}^{T} R=I$. Here, the index $\mu$ stands for multiplicity.

Variables, which are transformed and then assembled are denoted by $\widehat{w}$. We then have

$$
\widehat{w}:=R_{\mu}^{T} T^{T} w=R_{\mu}^{T} \bar{w} .
$$

By construction, $\widehat{w}$ is continuous for the a posteriori set of primal variables given by $\Pi$ and the a priori primal variables given by $\Pi^{\prime}$. The reasoning can be found in detail in the proof of Lemma 6.3; see Section 10.

We will describe all steps in detail and for general scalings. The subtle details appear in the proof of Theorem 6.7. Our results are therefore also of interest for the adaptive BDDC methods in $[1,7,31,54,55,63]$, which combine deluxe scaling with a transformation of basis.

For simplicity, we always assume we have the a priori coarse space with all a priori constraints enforced by partial assembly as in [39, 43, 44, 47]. Then, our a posteriori coarse space consisting of a posteriori constraints is implemented using a transformation of basis and partial assembly.

For the deflation approach, we introduce the finite element space

$$
\widetilde{W}_{\widehat{Q}}:=\left\{w \in \widetilde{W}: \widehat{Q}^{T} w=0\right\},
$$

where the constraint vectors are stored in the columns of $\widehat{Q}$. We typically have $\widehat{Q}=B^{T} \widehat{U}$, where $\widehat{U}$ contains the deflation vectors.

For our generalized transformation-of-basis approach, we introduce the space

$$
\widetilde{W}_{T, a}:=\left\{\widehat{w}=R_{\mu}^{T} T^{T} w: w \in \widetilde{W}\right\} .
$$

Here, certain columns in the transformation matrix $T$ represent the deflation vectors. The two spaces enforce the same constraints but correspond to different methods.

In the following, we will work with the space $\widetilde{W}_{T, a}$ where all variables are transformed to the new basis and are continuous in all primal variables. Let us recall that our orthogonal transformation $T^{T}$ will perform the change of basis from the standard nodal finite element basis to a non-nodal basis, e.g., with explicit averages or adaptive constraints. The inverse $T$ then transforms back to the nodal basis.

In subsequent sections, we just work with $B_{\Gamma}$ and $B_{D, \Gamma}$, and in order to simplify the notation, we write $B=B_{\Gamma}$ and $B_{D}=B_{D, \Gamma}$. In our theoretical considerations, we will use the space $\widetilde{W}_{\widehat{Q}}$. In our implementation, we iterate in the space $\widetilde{W}_{T, a}$. The space $\widetilde{W}_{T, a}$ is obtained via partial subassembly and scattering of the corresponding values.

Note that when using the generalized transformation-of-basis approach, for nodes with multiplicities larger than three, it can be simpler to implement constraints which can be stronger than the ones using deflation; cf. Figure 4.1. In this case, the condition number using the generalized transformation-of-basis approach is smaller than when using deflation. The coarse space size remains unchanged if the stronger constraints are chosen.

As motivated in the introduction and shown in the following sections, the construction of a transformation-of-basis approach with a posteriori constraints that yields the same condition number as the deflation approach requires some modifications of the theory compared to 
standard FETI-DP approaches, i.e., where only a priori constraints are used. This results from the fact that the primal components of $P_{D} w$ do in general not vanish-in contrast to standard theory; cf. the motivation in Section 4. In the following sections, we define the new $\widehat{P}_{D}$ operator and show the correspondence to a deflation method by replacing the standard arguments in question by those given in our Lemmas 6.3 and 6.4.

5.2. The generalized transformation-of-basis approach for FETI-DP. For transformed and assembled variables, we now define the transformed operators $\widehat{P}_{D}$ and $\widehat{\widetilde{S}}$ by

$$
\widehat{P}_{D}:=R_{\mu}^{T} T^{T} P_{D} T R \quad \text { and } \quad \widehat{\widetilde{S}}:=R^{T} T^{T} \widetilde{S} T R
$$

where $P_{D}=B_{D}^{T} B$. Note that the operator $P_{D}$ is built from the jump operator $B$ and the a priori scaling $D$ used with the a priori coarse space corresponding to the index set $\Pi^{\prime}$.

Let us recall that $R^{T} T^{T}$ and $R_{\mu}^{T} T^{T}$ map functions from $\widetilde{W}$ to $\widetilde{W}_{T, a}$, the space where the variables are transformed and assembled in all primal variables. The operator $T R$ then maps functions from $\widetilde{W}_{T, a}$ to the subspace $\widetilde{W}_{\widehat{Q}} \subset \widetilde{W}$, the space where only the a priori primal variables are assembled; cf. the lemmas in the following section.

For the theory, we will also use

$$
\widehat{B}:=B T R \quad \text { and } \quad \widehat{B}_{D}:=B_{D} T R_{\mu} .
$$

In practice, we will not implement a transformed version of $B$ or $B_{D}$ but only carry out the given matrix-vector multiplications before or after the (transposed) jump operator.

Let $\widetilde{S}$ be the Schur complement using the standard nodal basis and assembled only in the a priori set of primal variables. The operator $T^{T} \widetilde{S} T$ is then transformed to the non-nodal basis, e.g., based on edge averages or adaptive constraints, and $\widehat{\widetilde{S}}=R^{T} T^{T} \widetilde{S} T R$ is assembled also in the a posteriori primal variables such that the new constraints are enforced; see (5.3).

In our generalized transformation-of-basis approach, the transformed, preconditioned FETI-DP system matrix using the transformed Dirichlet preconditioner $\widehat{M}_{D}^{-1}$ is thus given by

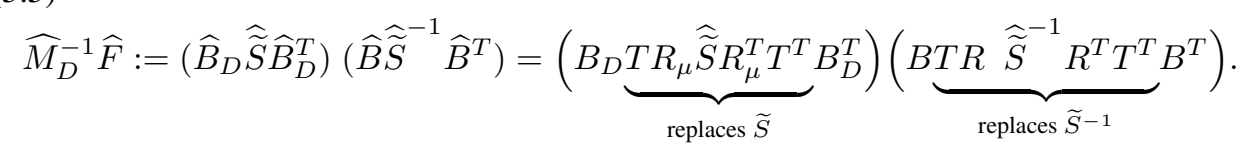

Here, $T R_{\mu} \widehat{\widetilde{S}} R_{\mu}^{T} T^{T}$ and $T R \widehat{\widetilde{S}}^{-1} R^{T} T^{T}$ replace the operators $\widetilde{S}$ and $\widetilde{S}^{-1}$ used in FETI-DP with deflation.

Note that the number of Lagrange multipliers and, thus, the number of rows in $B$ is the same as in the deflation approach since we keep Lagrange multipliers related to a posteriori primal variables in the system. This is the essential difference to the standard transformation-ofbasis approach, where $B_{\Delta}$ is used; see (5.6). This is possible since in the operator $R \widehat{\widetilde{S}}^{-1} R^{T}$, the multiplication with $R$ from the left does not change the a posteriori primal variables; the multiplication by $T$ in $T R \widehat{\widetilde{S}}^{-1} R^{T} T^{T}$ then transforms back to a standard nodal basis; as a result, the standard jump operator $B$ can be applied from the left.

For the case of nonredundant Lagrange multipliers, by [41, Theorem 6.5], we know that there exists a matrix $T_{\lambda}$ so that $B T=T_{\lambda} B$. In the next section, we will provide a lemma that extends [41, Theorem 6.5] to the case of redundant Lagrange multipliers. 
The preconditioned system (5.5) is different from the standard FETI-DP method using a transformation of basis as, e.g., in [39, 40, 41, 43, 47], which can be written using the transformed basis as

$$
M_{D}^{-1} F=\left(B_{D, \Delta} \widehat{\widetilde{S}} B_{D, \Delta}^{T}\right)\left(B_{\Delta} \widehat{\widetilde{S}}^{-1} B_{\Delta}^{T}\right)
$$

where the operator $B_{\Delta}$ only enforces continuity on the a posteriori dual variables and $B_{D, \Delta}$ is its scaled variant; cf., e.g., [44, 47].

5.3. Transformation in the space of Lagrange multipliers. A transformed scaling for FETI-DP can be defined by constructing a transformation of basis in the space of Lagrange multipliers as in [41]. This will, however, typically not be used in implementations. Instead the formulation given by (5.5) is implemented. This section is only provided for completeness; it can be skipped in a first reading.

Let us assume the case of redundant Lagrange multipliers and of transformation matrices $T^{(i)}, i=1, \ldots, N$, such that $T_{\mid \mathcal{Z}}^{(i)}=T_{\mid \mathcal{Z}}^{(s)}$ for any face or any edge $\mathcal{Z}$ and any adjacent pair $\left\{\Omega_{i}, \Omega_{s}\right\}, 1 \leq i, s \leq N$. Then, there exists a transformation of basis $T_{\lambda}$ in the space of Lagrange multipliers such that

$$
B T=T_{\lambda} B
$$

We also have

$$
B_{D} T=\left(D^{(1), T} T_{\lambda} B^{(1)}, \ldots, D^{(N), T} T_{\lambda} B^{(N)}\right) .
$$

For a proof, see [46]. A corresponding relationship was formulated for nonredundant Lagrange multipliers in [41, Theorem 6.5].

Now the transformed scaling can be defined. For a scaling matrix $D^{(i)}$, the explicit transformed scaling matrix $\widehat{D}^{(i)}$ is defined by

$$
\widehat{D}^{(i)}:=T_{\lambda}^{T} D^{(i)} T_{\lambda} \quad \text { for } \quad i=1, \ldots, N .
$$

For problems with constant coefficients on edges or faces, the transformed scaling remains diagonal if the original scaling was diagonal. For heterogeneous problems this is generally not the case.

6. Eigenvalues of FETI-DP with a generalized transformation-of-basis approach and with deflation or balancing. In this section, we show that FETI-DP using our generalized transformation-of-basis approach results in essentially the same eigenvalues as FETI-DP using the corresponding deflation or balancing approach. The generalized transformation-ofbasis approach is different from the standard FETI-DP and BDDC methods using a transformation of basis $[44,47,43,39]$ in that it allows for an interaction of dual and primal variables in the scaling.

In order to show the equivalence of eigenvalues, we need several assumptions collected here.

ASSUMPTION 1. First, we assume that a constraint vector in the deflation approach does not span several faces and/or edges. Second, if for an edge a constraint is enforced between two neighboring subdomain, then this constraint should be enforced between all neighboring subdomains, i.e., the situation given in Figure 4.1 (center) is not allowed. Third, but without loss of generality, we assume the numbering of nodes on the interface to be matching between adjacent subdomains. 


\section{ETNA}

Kent State University and

Johann Radon Institute (RICAM)

The first assumption is not valid for some FETI-DP methods given in the literature, e.g., for the FETI-DP method in [24] for almost incompressible elasticity, where all faces of each subdomain contribute only a single constraint. In this case, the generalized transformation-ofbasis approach cannot be used.

If the second assumption is not valid, then the generalized transformation-of-basis approach will result in a smaller condition number than when using the deflation approach. The coarse space size will remain unchanged, however.

REMARK 6.1. The generalized transformation-of-basis approach also results in the same number of zero eigenvalues as for FETI-DP using deflation (cf. Figure 8.3) and analogously in $(I-P)^{T} F U=0$ on range $\left(U^{T} F U\right)^{+}$; we also have $\widehat{F} U=0$.

To establish the equality of eigenvalues of FETI-DP (and BDDC) using the generalized transformation-of-basis approach and of FETI-DP using deflation, we will show that

$$
\left\langle\widehat{M}_{D}^{-1} \widehat{F} \widehat{\lambda}, \widehat{F} \widehat{\lambda}\right\rangle=\left\langle\widehat{P}_{D} \widehat{u}, \widehat{P}_{D} \widehat{u}\right\rangle_{\widehat{\widetilde{S}}}=\left\langle P_{D} u_{0}, P_{D} u_{0}\right\rangle_{\widetilde{S}}=\left\langle M_{P P}^{-1} F(I-P) \widehat{\lambda}, F(I-P) \widehat{\lambda}\right\rangle,
$$

where $\widehat{u} \in \widetilde{W}_{T, a}$ and $u_{0} \in \widetilde{W}_{\widehat{Q}}$; see Theorem 6.7.

For this, we will show that for any assembled and transformed vector $\widehat{w} \in \widetilde{W}_{T, a}$, we have a $w_{0}=T R \widehat{w} \in \widetilde{W}_{\widehat{Q}}$ such that

$$
\left|\widehat{P}_{D} \widehat{w}\right|_{\widetilde{S}}^{2}=\left|P_{D} w_{0}\right|_{\widetilde{S}}^{2}
$$

Vice versa, we will show that for any $w_{0} \in \widetilde{W}_{\widehat{Q}}$ a $\widehat{w}=R_{\mu}^{T} T^{T} w_{0} \in \widetilde{W}_{T, a}$ exists such that (6.1) holds, too.

We therefore have, for arbitrary scalings and coefficients, the same eigenvalues for the deflation approach and the corresponding generalized transformation-of-basis approach.

REMARK 6.2. In standard FETI-DP and BDDC theory, bounds of the form $\left|P_{D} w\right|_{\widetilde{S}}^{2} \leq C|w|_{\widetilde{S}}^{2}$ are established. For the adaptive coarse space approach in [32], we have $C=4 \max \left\{N_{\mathcal{F}}, N_{\mathcal{E}} M_{\mathcal{E}}\right\}^{2} \mathrm{TOL}$, where $N_{\mathcal{F}}$ denotes the maximum number of faces of any subdomain, $N_{\mathcal{E}}$ the maximum number of edges of any subdomain, $M_{\mathcal{E}}$ the maximum multiplicity of any edge, and TOL a given tolerance. For more details, we refer the reader to [33].

Using the definitions (5.2), (5.3), and (5.4), we have

$$
\begin{aligned}
\left.\left|\widehat{P}_{D} \widehat{w}\right|\right|_{\widetilde{S}} ^{2} & =\widehat{w}^{T}\left(\widehat{B}^{T} \widehat{B}_{D}\right) \widehat{\widetilde{S}}\left(\widehat{B}_{D}^{T} \widehat{B}\right) \widehat{w} \\
& =w^{T} T R_{\mu}\left(R^{T} T^{T} B^{T} B_{D} T R_{\mu}\right) R^{T} T^{T} \widetilde{S} T R\left(R_{\mu}^{T} T^{T} B_{D}^{T} B T R\right) R_{\mu}^{T} T^{T} w .
\end{aligned}
$$

Given $w_{0} \in \widetilde{W}_{\widehat{Q}}$, we would like to show that $T R R_{\mu}^{T} T^{T} B_{D}^{T} B w_{0}=B_{D}^{T} B w_{0}$. This, however, is not directly clear and is the subject of Lemma 6.3.

Classically, it is argued (see, e.g., [45, 47, 43, 62]) that the operator $T R R_{\mu}^{T} T^{T}$ reduces to the identity on the dual variables and that $B_{D}^{T} B w_{0}$ is zero in the primal variables. This latter argument, however, is not valid here since $B_{D}^{T} B w_{0}$ is not zero on the a posteriori set of primal variables if the generalized transformation-of-basis approach corresponding to the deflation approach is constructed. However, the newly introduced $\widehat{P}_{D}$ operator will be continuous in the a posteriori set of primal variables.

Lemma 6.3 essentially states that $T R R_{\mu}^{T} T^{T}$ can be seen as a projection onto the space span $\left\{B_{D}^{T} B w_{0}\right\}$ with $w_{0}$ given as before. In the following lemma, we also show the identity $T R R_{\mu}^{T} T^{T} w_{0}=w_{0}$, which will be of use in Lemma 6.4.

LEMMA 6.3. Given $w_{0} \in \widetilde{W}_{\widehat{Q}}$, we have

$$
T R R_{\mu}^{T} T^{T} w_{0}=w_{0} \quad \text { and } \quad T R R_{\mu}^{T} T^{T} B_{D}^{T} B w_{0}=B_{D}^{T} B w_{0} .
$$


For the proof, see Section 10. Let us now have a closer look at $\widehat{B} \widehat{w}=B T R R_{\mu}^{T} T^{T} w$.

LEMma 6.4. For $\widehat{w} \in \widetilde{W}_{T, a}$ there exists a $w_{0}:=T R \widehat{w} \in \widetilde{W}_{\widehat{Q}}$ with

$$
\widehat{B} \widehat{w}=B w_{0} .
$$

Vice versa, for $w_{0} \in \widetilde{W}_{\widehat{Q}}$ there exists a $\widehat{w}:=R_{\mu}^{T} T^{T} w_{0} \in \widetilde{W}_{T, a}$ satisfying (6.2).

For the proof, see Section 10. We now prove the main relation for the deflation or the balancing and the generalized transformation-of-basis approach; see equation (6.1).

LEMmA 6.5. For $\widehat{w} \in \widetilde{W}_{T, a}$ there exists a $w_{0}:=T R \widehat{w} \in \widetilde{W}_{\widehat{Q}}$ such that

$$
\left|\widehat{P}_{D} \widehat{w}\right|_{\widetilde{S}}^{2}=\left|P_{D} w_{0}\right|_{\widetilde{S}}^{2}
$$

holds. Vice versa, for $w_{0} \in \widetilde{W}_{\widehat{Q}}$ there exists a $\widehat{w}:=R_{\mu}^{T} T^{T} w_{0} \in \widetilde{W}_{T, a}$ such that (6.3) holds.

For the proof, see Section 10. We will now present a lemma essentially based on Lemma 6.4, Lemma 6.3, and [43, equation (8.1)].

Lemma 6.6. For $\widehat{w} \in \widetilde{W}_{T, a}$, we have $\widehat{B} \widehat{P}_{D} \widehat{w}=\widehat{B} \widehat{w}$.

For the proof, see Section 10. Note that Lemma 6.5 and Lemma 6.6 provide all the tools to prove identical condition numbers for FETI-DP using a generalized transformationof-basis approach and FETI-DP with deflation or balancing: From Lemma 6.5, we have $\left|\widehat{P}_{D} \widehat{w}\right|_{\widetilde{S}}^{2}=\left|P_{D} w_{0}\right|_{\widetilde{S}}^{2}$. The relation $|\widehat{w}|_{\widetilde{S}}=\left|w_{0}\right|_{\widetilde{S}}$ for $\widehat{w} \in \widetilde{W}_{T, a}$ and $w_{0} \in \widetilde{W}_{\widehat{Q}}$ can also be shown. The standard Rayleigh quotient estimate, e.g., [57, Theorem 2.4.2], [38, Lemma 3.2], and [43, Theorem 8.2] then gives the eigenvalue bound. However, with Theorem 6.7, we give a more general statement on the equality of all eigenvalues of the preconditioned operators, where the relation between $\left|w_{0}\right|_{\widetilde{S}}$ and $|\widehat{w}|_{\widetilde{S}}$ is not needed explicitly.

We can now formulate and prove the main theorem of our work.

THEOREM 6.7. Let an a priori coarse space ensure the invertibility of the local problems, e.g., defined by a sufficient number of primal vertices. Then,

$$
\sigma\left(\widehat{M}_{D}^{-1} \widehat{F}\right)=\sigma\left(M_{P P}^{-1} F\right),
$$

i.e., the eigenvalues of the preconditioned FETI-DP system matrix $\left(\widehat{M}_{D}^{-1} \widehat{F}\right)$ using a generalized transformation-of-basis approach are the same as for the preconditioned FETI-DP system matrix $\left(M_{P P}^{-1} F\right)$ using deflation.

Furthermore,

$$
\sigma\left(\widehat{M}_{D}^{-1} \widehat{F}\right) \backslash\{0\} \subset \sigma\left(M_{B P}^{-1} F\right),
$$

i.e., any nontrivial eigenvalue of the preconditioned FETI-DP system matrix $\left(\widehat{M}_{D}^{-1} \widehat{F}\right)$ using a generalized transformation-of-basis approach equals an eigenvalue of the preconditioned FETI-DP system matrix $\left(M_{B P}^{-1} F\right)$ using balancing.

Proof. For an arbitrary $\widehat{\lambda}$, we define $\widehat{u}:=\widehat{\widetilde{S}}^{-1} \widehat{B}^{T} \widehat{\lambda} \in \widetilde{W}_{T, a}$. Then, we have

$$
\left\langle\widehat{M}_{D}^{-1} \widehat{F} \widehat{\lambda}, \widehat{F} \widehat{\lambda}\right\rangle=\left\langle\widehat{B}_{D} \widehat{\widetilde{S}} \widehat{B}_{D}^{T} \widehat{B} \widehat{\widetilde{S}}^{-1} \widehat{B}^{T} \widehat{\lambda}, \widehat{B} \widehat{\widetilde{S}}^{-1} \widehat{B}^{T} \widehat{\lambda}\right\rangle=\left\langle\widehat{P}_{D} \widehat{u}, \widehat{P}_{D} \widehat{u}\right\rangle_{\widetilde{S}}
$$

as, e.g., in [43, Theorem 8.2]; cf. the Definitions in (5.5), (5.4), and (5.3).

With $u_{0}:=T R \widehat{u} \in \widetilde{W}_{\widehat{Q}}$ (cf. Lemma 6.4) consider

$$
\underbrace{R^{T} T^{T} \widetilde{S} T R}_{=\widehat{\widetilde{S}}} R_{\mu}^{T} T^{T} u_{0}=\widehat{\widetilde{S}} \widehat{u}=\widehat{B}^{T} \widehat{\lambda}=R^{T} T^{T} B^{T} \widehat{\lambda} .
$$


Now, we argue as in the proof of [41, Theorem 6.8] only that the operators are adapted slightly. So, equivalently to (6.5), we may solve the saddle point problem

$$
\left[\begin{array}{cc}
\widetilde{S} & \widehat{Q} \\
\widehat{Q}^{T} & 0
\end{array}\right]\left[\begin{array}{c}
u_{0} \\
\mu
\end{array}\right]=\left[\begin{array}{c}
B^{T} \widehat{\lambda} \\
0
\end{array}\right],
$$

where the assembly was replaced by the constraint $\widehat{Q}^{T} u_{0}=0$, i.e., we have $\widehat{Q}^{T} T R=0$, which explicitly uses the matrix $\widehat{Q}$ of $\widetilde{W}_{\widehat{Q}}:=\left\{w \in \widetilde{W}: \widehat{Q}^{T} w=0\right\}$. Note that this is connected to the deflation constraint matrix $\widehat{U}$ by $\widehat{Q}=B^{T} \widehat{U}$; see Section 5. From solving the saddle point system (6.6), we obtain with $\mu \in \operatorname{range}\left(\widehat{Q}^{T} \widetilde{S}^{-1} \widehat{Q}\right)^{+}$that

$$
\begin{aligned}
u_{0} & =\left(I-\widetilde{S}^{-1} \widehat{Q}\left(\widehat{Q}^{T} \widetilde{S}^{-1} \widehat{Q}\right)^{+} \widehat{Q}^{T}\right) \widetilde{S}^{-1} B^{T} \widehat{\lambda} \\
& =\left(I-\widetilde{S}^{-1} B^{T} \widehat{U}\left(\widehat{U}^{T} B \widetilde{S}^{-1} B^{T} \widehat{U}\right)^{+} \widehat{U}^{T} B\right) \widetilde{S}^{-1} B^{T} \widehat{\lambda}
\end{aligned}
$$

Thus, we obtain

$$
B u_{0}=\left(I-F \widehat{U}\left(\widehat{U}^{T} F \widehat{U}\right)^{+} \widehat{U}^{T}\right) F \widehat{\lambda}=(I-\widehat{P})^{T} F \widehat{\lambda}=(I-\widehat{P})^{T} F(I-\widehat{P}) \widehat{\lambda}
$$

with $\widehat{P}:=\widehat{U}\left(\widehat{U}^{T} F \widehat{U}\right)+\widehat{U}^{T} F$; see (3.5) and (3.7). Note that $\widehat{P}$ satisfies the same properties with respect to $\widehat{U}$ as $P$ with respect to $U$.

Using Lemma 6.5, (6.4), and (6.7), we obtain

$$
\begin{aligned}
\left\langle\widehat{M}_{D}^{-1} \widehat{F} \widehat{\lambda}, \widehat{F} \widehat{\lambda}\right\rangle & \stackrel{(6.4)}{=}\left\langle\widehat{P}_{D} \widehat{u}, \widehat{P}_{D} \widehat{u}\right\rangle_{\widehat{S}} \\
& \stackrel{\text { Lemma } 6.5}{=}\left\langle P_{D} u_{0}, P_{D} u_{0}\right\rangle_{\widetilde{S}} \stackrel{(3.8),(6.7)}{=}\left\langle M_{P P}^{-1} F(I-\widehat{P}) \widehat{\lambda}, F(I-\widehat{P}) \widehat{\lambda}\right\rangle .
\end{aligned}
$$

Then, using (6.8) and the Courant-Fischer-Weyl min-max principle, we obtain for the eigenvalues of $\widehat{M}_{D}^{-1} \widehat{F}$ and $M_{P P}^{-1} F$, the equality

$$
\begin{aligned}
\mu_{k}\left(\widehat{M}_{D}^{-1} \widehat{F}\right) & =\min _{\operatorname{dim}(V)=k} \max _{\widehat{\lambda} \in V:\|\widehat{\lambda}\|=1}\left\langle\widehat{M}_{D}^{-1} \widehat{F} \widehat{\lambda}, \widehat{F} \widehat{\lambda}\right\rangle \\
& =\min _{\operatorname{dim}(V)=k} \max _{\widehat{\lambda} \in V:\|\widehat{\lambda}\|=1}\left\langle M_{P P}^{-1} F(I-P) \widehat{\lambda}, F(I-P) \widehat{\lambda}\right\rangle=\mu_{k}\left(M_{P P}^{-1} F\right),
\end{aligned}
$$

where $\mu_{k}\left(\widehat{M}_{D}^{-1} \widehat{F}\right)$ and $\mu_{k}\left(M_{P P}^{-1} F\right)$ denote the respective $k$-th eigenvalue sorted in increasing order. The relation between the eigenvalues of $M_{P P}^{-1} F$ and $M_{B P}^{-1} F$ can be found in [51] or, using our notation, in [41].

Note that we have $0 \in \sigma\left(\widehat{M}_{D}^{-1} \widehat{F}\right)$ also for the case of nonredundant Lagrange multipliers if $\widehat{U}$ is not empty; cf. Remark 6.1. This is different compared to the classic FETI-DP methods using a transformation of basis and results from the fact that constraints in $B$ are applied to vectors which are already continuous in the a posteriori primal variables. These Lagrange multipliers are not discarded since they allow us to implement an interaction of a posteriori primal and a posteriori dual variables through the scaling in $B_{D}$; see the preconditioned system in (5.5).

7. Modified operators and condition number estimate for BDDC with a generalized transformation-of-basis approach. In the previous sections, we have shown that we can use the generalized transformation-of-basis in order to derive a FETI-DP approach using a change of variables and a partial assembly with the same condition number as a corresponding FETI-DP method with the deflation or the balancing approach. Given the close relations 
between FETI-DP and BDDC methods, a corresponding BDDC method using a generalized transformation-of-basis approach can also be constructed.

We will use the assembly operator $R_{\Delta^{\prime}}^{T}$ that assembles all degrees of freedom of $\Delta^{\prime}=\Pi \cup \Delta$, i.e., all a posteriori primal $(\Pi)$ and remaining dual $(\Delta)$ degrees of freedom; cf. the presentation of standard BDDC at the end of Section 3.1. Then, we introduce the notation

$$
R^{\prime}:=\left[\begin{array}{cc}
I_{\Pi^{\prime}} & 0 \\
0 & R_{\Delta^{\prime}}
\end{array}\right]
$$

where $R^{\prime}$ leaves the initial coarse space variables unchanged and performs the assembly in all other interface variables. The BDDC system matrix is thus the Schur complement on the interface

$$
\mathcal{S}=R^{\prime T} \widetilde{S} R^{\prime}
$$

Note that since the transformations are chosen consistently for any face and any edge and since $R^{\prime} R^{\prime T}$ assembles and redistributes information in both the a posteriori primal and the remaining dual degrees of freedom, we have

$$
T R^{\prime} R^{\prime T}=R^{\prime} R^{\prime T} T \quad \text { and } \quad T^{T} R^{\prime} R^{\prime T}=R^{\prime} R^{\prime T} T^{T} .
$$

We now introduce the scaling matrix $D_{u}$ for the untransformed degrees of freedom $u$ in BDDC corresponding to the untransformed scaling $D$ of the Lagrange multipliers in FETI-DP.

Definition 7.1 (Transformed Degree of Freedom Scaling). For a scaling matrix $D_{u}^{(i)}$, the transformed scaling matrix $\widehat{D}_{u}^{(i)}$ is defined by

$$
\widehat{D}_{u, \Delta^{\prime}}^{(i)}:=T_{\Delta^{\prime}}^{(i) T} D_{u, \Delta^{\prime}}^{(i)} T_{\Delta^{\prime}}^{(i)} \quad \text { for } i=1, \ldots, N .
$$

The transformed BDDC scaling is then given by $\widehat{D}_{u}:=T^{T} D_{u} T$. Note that, an identity is introduced in the scaling for the a priori primal variables $\Pi^{\prime}$ to fit the dimension in the equations; see the definition of $T$ in (10.6). Then, the BDDC preconditioner for the system matrix (7.2) is defined by

$$
\widehat{M}_{\mathrm{BDDC}}^{-1}:=R^{\prime T} D_{u} T R\left(R^{T} T^{T} \widetilde{S} T R\right)^{-1} R^{T} T^{T} D_{u} R^{\prime}=R^{\prime T} T \widehat{D}_{u} R \widehat{\widetilde{S}}^{-1} R^{T} \widehat{D}_{u} T^{T} R^{\prime},
$$

where $R^{\prime}$ was introduced in (7.1) and $R$ defined in (10.8) replicates the a posteriori primal variables.

Thus, the preconditioned system matrix in our generalized transformation-of-basis approach is

$$
\widehat{M}_{\mathrm{BDDC}}^{-1} \mathcal{S}=\left(R^{\prime T} T \widehat{D}_{u} R \widehat{\widetilde{S}}^{-1} R^{T} \widehat{D}_{u} T^{T} R^{\prime}\right)\left(R^{\prime T} \widetilde{S} R^{\prime}\right)
$$

Since the scaling $\widehat{D}_{u}$ affects dual and a posteriori primal variables, the method is clearly different from BDDC with a standard transformation of basis and transformed scaling, which can be written as

$$
M_{\mathrm{BDDC}}^{-1} \mathcal{S}=\left[\begin{array}{cc}
I_{\Pi \prime} \cup \Pi & 0 \\
0 & R_{\Delta, \widehat{D}_{u, \Delta}}^{T}
\end{array}\right] \widehat{\widetilde{S}}^{-1}\left[\begin{array}{cc}
I_{\Pi^{\prime} \cup \Pi} & 0 \\
0 & R_{\Delta, \widehat{D}_{u, \Delta}}
\end{array}\right] \mathcal{S}
$$


and where $\widehat{D}_{u, \Delta}$ is a transformed scaling acting only on the remaining dual variables $\Delta$ and $I_{\Pi \prime} \cup \Pi$ is the identity on all primal variables $\Pi^{\prime} \cup \Pi$. In our preconditioner, however, an interaction between a posteriori dual and primal variables can be implemented by using a nondiagonal $D_{u}$. This interaction can be necessary; cf. Section 4.2.

The $E_{D_{u}}$ operator, which is central to the condition number proof of BDDC, is given by $E_{D_{u}}:=R^{\prime} R^{\prime T} D_{u}$. We now define

$$
\widehat{E}_{D_{u}}:=R_{\mu}^{T} T^{T} R^{\prime} R^{\prime T} D_{u} T R
$$

LEMMA 7.2. We have

$$
\begin{aligned}
\widehat{E}_{D_{u}} & =R_{\mu}^{T} E_{\widehat{D}_{u}} R \\
\widehat{P}_{D} & =I-\widehat{E}_{D_{u}} .
\end{aligned}
$$

Proof.

i) By (7.3), we obtain

$$
\widehat{E}_{D_{u}}=R_{\mu}^{T} T^{T} R^{\prime} R^{\prime T} D_{u} T R=R_{\mu}^{T} R^{\prime} R^{\prime T} T^{T} D_{u} T R=R_{\mu}^{T} R^{\prime} R^{\prime T} \widehat{D}_{u} R=R_{\mu}^{T} E_{\widehat{D}_{u}} R .
$$

ii) Since $R_{\mu}^{T}=\left(R^{T} R\right)^{-1} R^{T}$, we have $R_{\mu}^{T} R=I$. Combining the previous statement, the standard relation $P_{D}=I-E_{D_{u}}$, and (5.3), we also have

$$
\widehat{P}_{D}=R_{\mu}^{T} T^{T} P_{D} T R=R_{\mu}^{T}\left(I-E_{\widehat{D}_{u}}\right) R=I-\widehat{E}_{D_{u}} .
$$

THEOREM 7.3. Let an a priori coarse space ensure the invertibility of the local problems, e.g., defined by using a sufficient number of primal vertices. Then

$$
\sigma\left(\widehat{M}_{B D D C}^{-1} \mathcal{S}\right) \backslash\{0,1\} \subset \sigma\left(\widehat{M}^{-1} \widehat{F}\right)=\sigma\left(M_{P P}^{-1} F\right),
$$

i.e., except for zeros and ones, the preconditioned BDDC system matrix $\widehat{M}_{B D D C}^{-1} \mathcal{S}$ has the same eigenvalues as the preconditioned FETI-DP system matrix using either a generalized transformation-of-basis approach or deflation.

Proof. The proof is based on the known relation between BDDC and FETI-DP; see [47]. The preconditioned BDDC system operator is given by

$$
\widehat{M}_{\mathrm{BDDC}}^{-1} \mathcal{S}=\left(R^{\prime T} T \widehat{D}_{u} R \widehat{\widetilde{S}}^{-1} R^{T} \widehat{D}_{u} T^{T} R^{\prime}\right)\left(R^{\prime T} \widetilde{S} R^{\prime}\right),
$$

which, except for zeros, has the same eigenvalues as

$$
\widehat{\widetilde{S}}^{-1} R^{T} \widehat{D}_{u} T^{T} R^{\prime} R^{\prime T} \widetilde{S} R^{\prime} R^{\prime T} T \widehat{D}_{u} R
$$

From (7.3) and $R R_{\mu}^{T} R^{\prime}=R^{\prime}$, we obtain

$$
\begin{aligned}
\widehat{\widetilde{S}}^{-1} R^{T} \widehat{D}_{u} T^{T} R^{\prime} R^{\prime T} \widetilde{S} R^{\prime} R^{\prime T} T \widehat{D}_{u} R & =\widehat{\widetilde{S}}^{-1} R^{T} \widehat{D}_{u} R^{\prime} R^{\prime T} R_{\mu} R^{T} T^{T} \widetilde{S} T R R_{\mu}^{T} R^{\prime} R^{\prime T} \widehat{D}_{u} R \\
& =\widehat{\widetilde{S}}^{-1} \widehat{E}_{D_{u}}^{T} \widehat{\widetilde{S}} \widehat{E}_{D_{u}},
\end{aligned}
$$

which then has the same eigenvalues as

$$
\widehat{E}_{D_{u}} \widehat{\widetilde{S}}^{-1} \widehat{E}_{D_{u}}^{T} \widehat{\widetilde{S}}
$$

By using $\widehat{P}_{D}=I-\widehat{E}_{D_{u}}$ from Lemma 7.2 and the estimate from Theorem 6.7, we find that the eigenvalues (except for zero and one) of the BDDC method using the generalized transformation-of-basis approach are identical to those of FETI-DP using the generalized transformation-of-basis or the deflation approach. 


\section{ETNA}

Kent State University and Johann Radon Institute (RICAM)
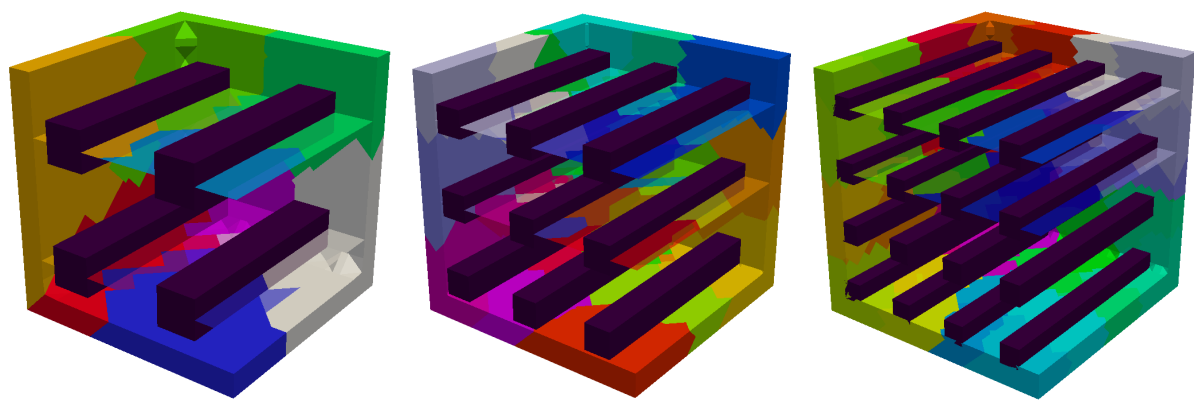

FIG. 8.1. Irregular decomposition of the unit cube with a composite material using METIS [28, 29]. High coefficients $\rho_{2}=1 e+06$ are shown in dark purple in the picture, and subdomains are shown in different colors in the background and by half-transparent slices. Visualization for $N=8$ subdomains and $1 / h=12$ (left), $N=27$ subdomains and $1 / h=18$ (center), and $N=64$ subdomains and $1 / h=24$ (right).
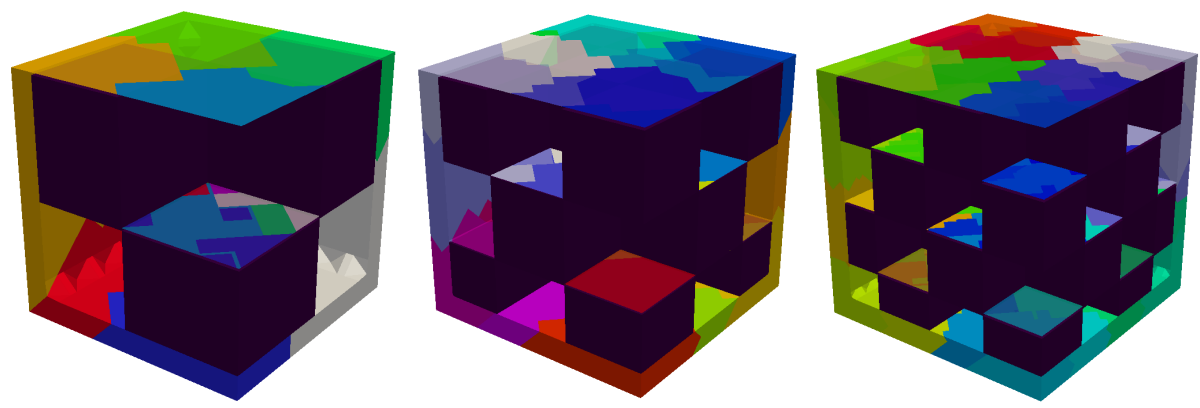

FIG. 8.2. Irregular decomposition of the unit cube with a regular checkerboard material using METIS [28, 29]. High coefficients $\rho_{2}=1 e+06$ are shown in dark purple in the picture, and are subdomains shown in different colors in the background and by half-transparent slices. Visualization for $N=8$ subdomains and $1 / h=12$ (left), $N=27$ subdomains and $1 / h=18$ (center), and $N=64$ subdomains and $1 / h=24$ (right).

Remarks on the implementation of BDDC. Let us note that as in the case of adaptive FETI-DP, the a posteriori set of primal degrees of freedom (given by the index set $\Pi$ ) also have to be scaled by the transformed scaling $\widehat{D}_{u}$. Thus, compared to the standard BDDC preconditioner, we replace $\widetilde{S}^{-1}$ by $\widehat{\widetilde{S}}^{-1}, D_{u}$ by $\widehat{D}_{u}$ and assemble, using $R^{T}$, the a posteriori primal degrees of freedom between the application of the scaling $\widehat{D}_{u}$ and the solution of the system of equations associated with $\widehat{\widetilde{S}}$, i.e., in the preconditioner we solve systems of the form $\widehat{\widetilde{S}} x=R^{T} \widehat{D}_{u} w$ for the unknown $x \in \widetilde{W}_{T, a}$; see (7.4).

8. Application to an example for the diffusion equation. We now present results for the diffusion equation with highly varying coefficients $\rho \in[1,1 \mathrm{e}+06]$ on the unit cube $\Omega=[0,1]^{3}$ and an irregular METIS (see [28, 29]) decomposition for $N$ subdomains. For the face with $x=0$, we enforce homogeneous Dirichlet boundary conditions and for all other faces homogeneous Neumann boundary conditions. We consider two materials. First, we consider a soft matrix material with $\rho_{1}=1$ and an embedded stiff material in the form of $N^{2 / 3}$ beams with $\rho_{2}=1 \mathrm{e}+06$ running from the face with $x=0$ to the face with $x=1$; see Figure 8.1. In the second example, the Young modulus is distributed in a regular checkerboard pattern; see Figure 8.2. We apply the adaptive coarse space approach from [32] to obtain a method which is independent of the coefficient jump. Our convergence criterion for the preconditioned conjugate gradients is a relative reduction of the preconditioned residual of $1 \mathrm{e}+06$. Our results 


\section{ETNA}

Kent State University and

Johann Radon Institute (RICAM)

TABLE 8.1

Diffusion equation with $\rho_{1}=1, \rho_{2}=1 e+06$. Coarse spaces for TOL $=10$ for all generalized eigenvalue problems. $\left|\Pi^{\prime}\right|$ : size of a priori coarse space, $|\Pi|$ : number of additional a posteriori constraints in the generalized transformation-of-basis approach (gToB), $|\widehat{U}|$ : number of additional constraints in the deflation approach.

3D Composite material ( $N^{2 / 3}$ beams), METIS partitioning, and $1 / h=6 N^{1 / 3}$.

\begin{tabular}{c|c|c|c|c|c|c|c|c|c|c|c|c|c}
\hline \multicolumn{4}{|c|}{ FETI-DP $($ Deflation/PP) } & \multicolumn{4}{c|}{ FETI-DP $(\mathrm{gToB})$} & \multicolumn{4}{|c}{ BDDC $(\mathrm{gToB})$} \\
\hline$N$ & $\left|\Pi^{\prime}\right|$ & $\lambda_{\min }$ & $\lambda_{\max }$ & its & $|\widehat{U}|$ & $\lambda_{\min }$ & $\lambda_{\max }$ & its & $|\Pi|$ & $\lambda_{\min }$ & $\lambda_{\max }$ & its & $|\Pi|$ \\
\hline $2^{3}$ & 30 & 1.00 & $\mathbf{7 . 5 9}$ & 14 & 32 & 1.00 & $\mathbf{7 . 5 9}$ & 14 & 20 & 1.00 & $\mathbf{7 . 5 9}$ & 13 & 20 \\
$3^{3}$ & 165 & 1.00 & $\mathbf{8 . 1 9}$ & 18 & 203 & 1.00 & $\mathbf{8 . 1 9}$ & 18 & 135 & 1.00 & $\mathbf{8 . 1 9}$ & 14 & 135 \\
$4^{3}$ & 468 & 1.00 & $\mathbf{1 0 . 2 7}$ & 23 & 545 & 1.00 & $\mathbf{1 0 . 2 7}$ & 23 & 336 & 1.00 & $\mathbf{1 0 . 2 7}$ & 18 & 336 \\
$5^{3}$ & 1066 & 1.00 & $\mathbf{1 0 . 8 8}$ & 23 & 1071 & 1.00 & $\mathbf{1 0 . 8 8}$ & 22 & 645 & 1.00 & $\mathbf{1 0 . 8 8}$ & 18 & 645 \\
$6^{3}$ & 1878 & 1.00 & $\mathbf{9 . 2 0}$ & 23 & 1837 & 1.00 & $\mathbf{9 . 2 0}$ & 23 & 1099 & 1.00 & $\mathbf{9 . 2 0}$ & 18 & 1099
\end{tabular}

TABLE 8.2

Diffusion equation with $\rho_{1}=1, \rho_{2}=1 e+06$. Coarse spaces for TOL $=10$ for all generalized eigenvalue problems. $\left|\Pi^{\prime}\right|$ : size of a priori coarse space, $|\Pi|$ : number of additional a posteriori constraints in the generalized transformation-of-basis approach $(g T o B),|\widehat{U}|:$ number of additional constraints in the deflation approach.

3D checkerboard coefficients ( $\lceil N / 2\rceil$ cubes with high coefficients), METIS partitioning, and $1 / h=6 N^{1 / 3}$.

\begin{tabular}{c|c|c|c|c|c|c|c|c|c|c|c|c|c}
\hline \multicolumn{2}{c|}{} & \multicolumn{3}{|c|}{ FETI-DP (Deflation/PP) } & \multicolumn{4}{c|}{ FETI-DP $(\mathrm{gToB})$} & \multicolumn{4}{c}{ BDDC $(\mathrm{gToB})$} \\
\hline$N$ & $\left|\Pi^{\prime}\right|$ & $\lambda_{\min }$ & $\lambda_{\max }$ & its & $|\widehat{U}|$ & $\lambda_{\min }$ & $\lambda_{\max }$ & its & $|\Pi|$ & $\lambda_{\min }$ & $\lambda_{\max }$ & its & $|\Pi|$ \\
\hline $2^{3}$ & 30 & 1.00 & $\mathbf{7 . 3 1}$ & 17 & 14 & 1.00 & $\mathbf{7 . 3 1}$ & 17 & 9 & 1.00 & $\mathbf{7 . 3 1}$ & 14 & 9 \\
$3^{3}$ & 165 & 1.00 & $\mathbf{8 . 3 5}$ & 20 & 45 & 1.00 & $\mathbf{8 . 3 5}$ & 20 & 29 & 1.00 & $\mathbf{8 . 3 5}$ & 16 & 29 \\
$4^{3}$ & 468 & 1.00 & $\mathbf{8 . 9 3}$ & 22 & 188 & 1.00 & $\mathbf{8 . 9 3}$ & 22 & 120 & 1.00 & $\mathbf{8 . 9 3}$ & 18 & 120 \\
$5^{3}$ & 1066 & 1.00 & $\mathbf{1 2 . 3 6}$ & 22 & 245 & 1.00 & $\mathbf{1 2 . 3 6}$ & 22 & 150 & 1.00 & $\mathbf{1 2 . 3 6}$ & 18 & 150 \\
$6^{3}$ & 1878 & 1.00 & $\mathbf{9 . 7 2}$ & 23 & 545 & 1.00 & $\mathbf{9 . 7 2}$ & 23 & 326 & 1.00 & $\mathbf{9 . 7 2}$ & 19 & 326
\end{tabular}

in Tables 8.1 and 8.2 show identical estimates for $\lambda_{\min }$ and $\lambda_{\max }$ for all three methods in accordance with the theory. In Figure 8.3, all eigenvalues of the three preconditioned operators were computed numerically for $1 / h=12$. We see that indeed all eigenvalues other than 0 and 1 are identical, as predicted by the theory. Note that the sizes of $|\Pi|$ and $|\widehat{U}|$ are not equal. An explanation is given in Section 4 and Figure 4.1.

9. Conclusion. The focus of this paper is to provide a generalized transformation-ofbasis approach for FETI-DP and BDDC with essentially the same eigenvalues as known FETI-DP methods using deflation or balancing.

A known disadvantage of deflation and balancing methods is that the coarse space has to be solved quite exactly; cf. [41]. The generalized transformation-of-basis approach or the use of local saddle point problems provide a robust remedy, and adaptive multi-level extensions are easier to construct.

We have presented FETI-DP and BDDC methods using the deflation vectors in the construction of the generalized transformation-of-basis approach; see (5.5) for FETI-DP and see (7.4) for BDDC. As in [41], we have to assume that the deflation vectors do not span several edges or faces.

For general scalings (as necessary for heterogeneous problems), the classical approaches to FETI-DP and BDDC with a standard transformation of basis have to be revisited and modified. The modifications result from the fact that in deflation or balancing, in general an interaction between primal and dual variables can occur. This interaction is not present in traditional FETI-DP and BDDC with a transformation of basis but is possible in our generalized approach. As a result of this interaction, a standard assumption, i.e., that $P_{D} w$ and $\left(I-E_{D_{u}}\right) w$ are zero in the second set of primal variables (a posteriori coarse space), is not valid anymore. In our theory, this traditional argument is replaced by Lemma 6.3. Building on 


\section{ETNA}

Kent State University and Johann Radon Institute (RICAM)
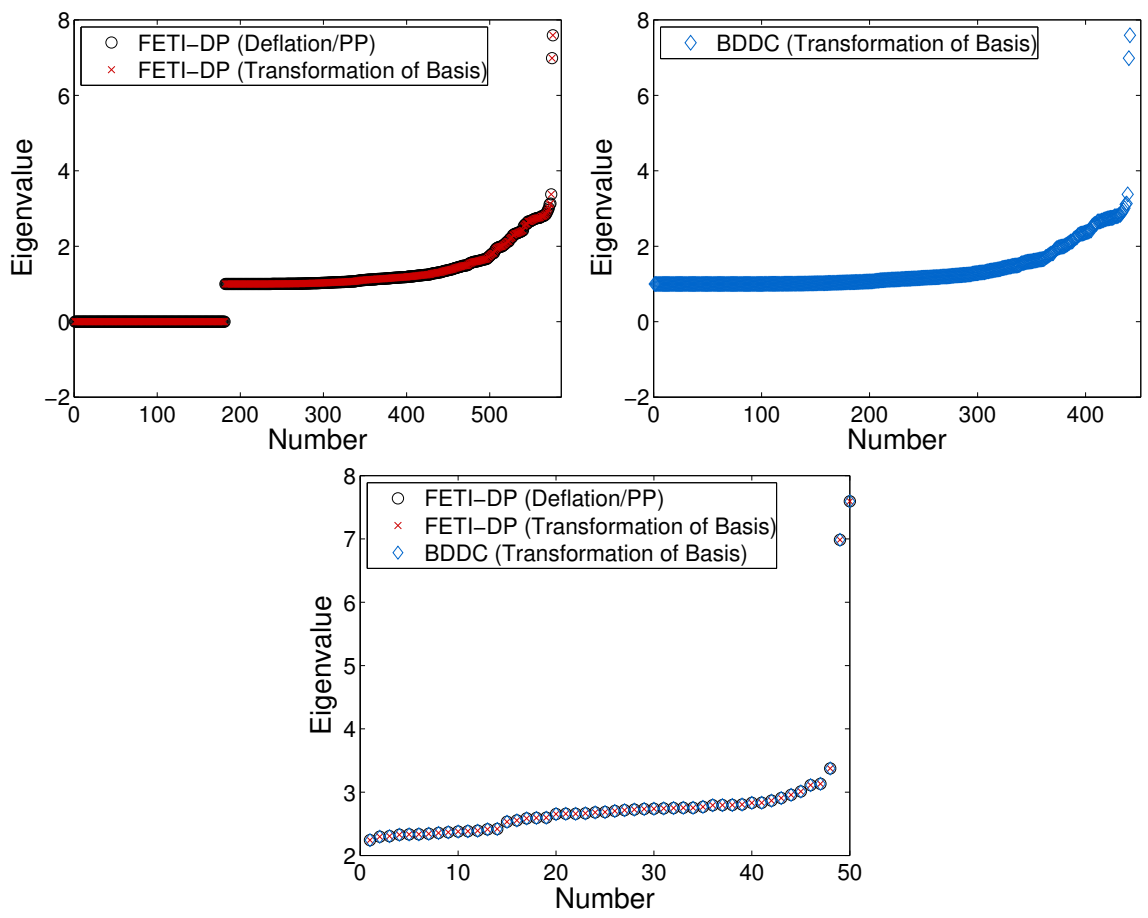

FIG. 8.3. Plot of the eigenvalues of the preconditioned operators for FETI-DP with projector preconditioning $\left(M_{P P}^{-1} F\right)$ and a transformation of basis $\left(\widehat{M}_{T}^{-1} \widehat{F}\right)$ (top left), BDDC with a transformation of basis $\left(\widehat{M}_{B D D C}^{-1} \mathcal{S}\right)($ top right) and the largest 50 eigenvalues of the preconditioned operators (bottom center) for the composite material, an irregular decomposition of the unit cube into eight subdomains, and $1 / h=12$. The eigenvalues greater than one are identical for all three algorithms.

this lemma, the equivalence between the generalized transformation-of-basis approach and the corresponding deflation or balancing approach can be shown.

Thus, finally, our FETI-DP and BDDC methods using a generalized transformationof-basis approach satisfy the same condition number bound and essentially have the same eigenvalues as the FETI-DP method using deflation or balancing. The numerical results presented for scalar elliptic problems support our findings.

In combination with adaptive coarse spaces, competitive parallel FETI-DP methods can be implemented using the generalized transformation-of-basis approach. For heterogeneous problems, these can improve the standard FETI-DP both with respect to time to solution and precision; see $[33,35,46]$.

10. Proofs of Lemma 6.3-Lemma 6.6. We here provide the proofs not given above.

Proof of Lemma 6.3. For the proof of this lemma, we exploit the structure of the given operators, which are block diagonal with respect to the local faces and edges. In order to facilitate the analysis, let us assume that a posteriori constraints are only associated with the edge $\mathcal{Z}_{l_{1}}$. Now consider the orthonormalized set of constraint vectors $\left(q_{\mathcal{Z}_{l_{1}}}^{1}, \ldots, q_{\mathcal{Z}_{l_{1}}}^{r}\right)$ on $\mathcal{Z}_{l, 1}$. Then, introduce

$$
T_{\mathcal{Z}_{l_{1}}, \Pi_{\mathcal{Z}_{l_{1}}}}:=\left[q_{\mathcal{Z}_{l_{1}}}^{1}, \ldots, q_{\mathcal{Z}_{l_{1}}}^{r}\right] .
$$

Using a modified Gram-Schmidt algorithm, we compute a matrix $T_{\mathcal{Z}_{l_{1}}, \Delta_{\mathcal{Z}_{l_{1}}}}$ so that $T_{\mathcal{Z}_{l_{1}}}:=\left(T_{\mathcal{Z}_{l_{1}}, \Pi_{\mathcal{Z}_{l_{1}}}}, T_{\mathcal{Z}_{l_{1}}, \Delta_{\mathcal{Z}_{l_{1}}}}\right)$ is a square matrix and $T_{\mathcal{Z}_{l_{1}}}^{T} T_{\mathcal{Z}_{l_{1}}}=I$, i.e., $T_{\mathcal{Z}, \Delta_{\mathcal{Z}}}$ will 
be orthogonal to the constraint space $\operatorname{span}\left(q_{\mathcal{Z}_{l_{1}}}^{1}, \ldots, q_{\mathcal{Z}_{l_{1}}}^{r}\right)$. For convenience, we order the primal variables first.

For each subdomain $\Omega_{l}$, we denote the faces and/or edges by $\mathcal{Z}_{l_{1}}, \ldots, \mathcal{Z}_{l_{s}}$. For $n>1$, the matrix $T_{\mathcal{Z}_{l_{n}}, \Pi_{\mathcal{Z}_{l_{n}}}}$ is void and $T_{\mathcal{Z}_{l_{n}}}=\left[T_{\mathcal{Z}_{l_{n}}, \Delta_{\mathcal{Z}_{l_{n}}}}\right]=I$. We assume that the degrees of freedom of all the faces and edges of $\Omega_{l}$ are ordered such that the degrees of freedom on $\mathcal{Z}_{l_{1}}$ are ordered first, those of $\mathcal{Z}_{l_{2}}$ are ordered second, etc. Then

$$
T_{\Delta_{l}^{\prime}}^{(l)}:=\left(T_{\Pi_{l}}^{(l)}, T_{\Delta_{l}}^{(l)}\right):=\left[\begin{array}{cccccc}
T_{\mathcal{Z}_{l_{1}}, \Pi_{\mathcal{Z}_{l_{1}}}} & T_{\mathcal{Z}_{l_{1}}, \Delta_{\mathcal{Z}_{l_{1}}}} & 0 & \ldots & \ldots & 0 \\
0 & 0 & I & 0 & \ldots & 0 \\
\vdots & \vdots & 0 & \ddots & \ddots & \vdots \\
\vdots & \vdots & \vdots & \ddots & \ddots & 0 \\
0 & 0 & 0 & \ldots & 0 & I
\end{array}\right]
$$

represents the transformation from the new (non-nodal) basis to the old (nodal) basis, still missing an assembly operation, i.e.,

$$
w_{\Delta_{l}^{\prime}}^{(l)}=T_{\Delta_{l}^{\prime}}^{(l)} \bar{w}_{\Delta_{l}^{\prime}}^{(l)} .
$$

As mentioned before, the transformations are chosen consistently, i.e., for the three subdomains, we have for the local transformations of $\mathcal{Z}_{1}$ shared by $\Omega_{i}, \Omega_{j}$, and $\Omega_{k}$ that

$$
T_{\mathcal{Z}_{i_{1}}, \Pi_{\mathcal{Z}_{i_{1}}}}=T_{\mathcal{Z}_{j_{1}}, \Pi_{\mathcal{Z}_{j_{1}}}}=T_{\mathcal{Z}_{k_{1}}, \Pi_{\mathcal{Z}_{k_{1}}}} \text { and } T_{\mathcal{Z}_{i_{1}}, \Delta \mathcal{Z}_{i_{1}}}=T_{\mathcal{Z}_{j_{1}}, \Delta \mathcal{z}_{j_{1}}}=T_{\mathcal{Z}_{k_{1}}, \Delta \mathcal{Z}_{k_{1}}}
$$

$T_{\Delta_{l}^{\prime}}^{(l) T} T_{\Delta_{l}^{\prime}}^{(l)}=I, l \in\{i, j, k\}$, also holds, and the columns of $T_{\Pi_{l}}^{(l)}$ span the range of all constraint vectors associated with $\Omega_{l}, l \in\{i, j, k\}$. Therefore, using (10.2) and (10.3), we have

$$
T_{\Pi_{l}}^{(l) T} w_{l, \Delta_{l}^{\prime}}=\left[\begin{array}{llll}
I & 0 & \ldots & 0
\end{array}\right]\left[\begin{array}{c}
\bar{w}_{l, \Pi_{z_{l_{1}}}} \\
\bar{w}_{l, \Delta z_{l_{1}}} \\
\vdots \\
\bar{w}_{l, \Delta_{z_{l}}}
\end{array}\right]=\bar{w}_{l, \Pi_{\mathcal{z}_{l_{1}}}}, \quad \text { for } l \in\{i, j, k\} .
$$

Next, we can give the relation of the local transformation matrices and the global transformation matrix

$$
T=\left[\begin{array}{cc}
I_{\Pi^{\prime}} & 0 \\
0 & \text { blockdiag }_{l=1, \ldots, N}\left(T_{\Delta_{l}^{\prime}}^{(l)}\right)
\end{array}\right] .
$$

The transformed variables then still lack an assembly operation. In the following, we also use the simplified index $\mathcal{Z}_{1}$ instead of $\mathcal{Z}_{l_{1}}$, for $l \in\{i, j, k\}$, since this edge is shared by these three subdomains and since (10.4) holds. In order to enforce

$$
\bar{w}_{\Pi_{\mathcal{Z}_{1}}}^{(i)}=\bar{w}_{\Pi_{\mathcal{Z}_{1}}}^{(j)}=\bar{w}_{\Pi_{\mathcal{Z}_{1}}}^{(k)},
$$

we have introduced the global assembly operator $R^{T}$ and its transpose, the restriction operator $R$, which replicates the a posteriori primal degrees of freedom (given by the index set $\Pi$ ).

The restriction operator $R$ is of the form

$$
R=\left[\begin{array}{cccccc}
I_{\Pi^{\prime}} & 0 & 0 & \ldots & \ldots & 0 \\
0 & (*)_{\Pi_{1}} & (*)_{\Delta_{1}} & 0 & \ldots & 0 \\
\vdots & \vdots & 0 & \ddots & & \vdots \\
\vdots & \vdots & \vdots & & \ddots & 0 \\
0 & (*)_{\Pi_{N}} & 0 & \ldots & 0 & (*)_{\Delta_{N}}
\end{array}\right]
$$


where the matrix $\left((*)_{\Pi_{i}},(*)_{\Delta_{i}}\right), i=1, \ldots, N$, is a permutation of the columns of the identity matrix. The operator $R$ replicates the a posteriori degrees of freedom to the different subdomains but does not change the a priori set of primal variables.

The local version of $R^{T}$, restricted to the considered edge $\mathcal{Z}_{1}$, is given by

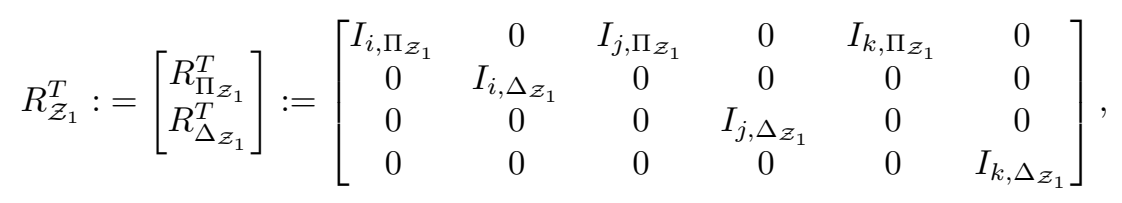

and the local version of the multiplicity-weighted operator is

$$
R_{\mathcal{Z}_{1}, \mu}^{T}:=\left[\begin{array}{c}
\frac{1}{3} R_{\Pi_{\mathcal{Z}_{1}}}^{T} \\
R_{\Delta_{\mathcal{Z}_{1}}}^{T}
\end{array}\right] .
$$

We are now ready to localize the expression $T R R_{\mu}^{T} T^{T}$ to prove the statements of the lemma.

In the following, we use $u \in\left\{w_{0}, B_{D}^{T} B w_{0}\right\}$ in order to realize certain computations for $w_{0}$ and $B_{D}^{T} B w_{0}$ simultaneously; $u$ will be replaced by the corresponding function when necessary.

First, consider $T R R_{\mu}^{T} T^{T} u$. From $u^{T}=\left(u^{(1) T}, \ldots, u^{(N) T}\right)^{T}$, we obtain the local functions $u^{(l)} \in W_{l}, l=1, \ldots, N$, and for $l \in\{i, j, k\}$, we define $u_{\mathcal{Z}_{1}}^{(l)}$ as the values at the degrees of freedom on the edge $\mathcal{Z}_{1}$. For $l=\{i, j, k\}$, the values of the local function $u^{(l)}$ on all remaining degrees of freedom on $\left(\partial \Omega_{l, h} \cap \Gamma_{h}\right) \backslash \mathcal{Z}_{1}$ are denoted by $u_{\mathcal{Z}_{1}^{C}}^{(l)}$. For $l \notin\{i, j, k\}$, we have $u^{(l)}=u_{\mathcal{Z}_{1}^{C}}^{(l)}$.

Thus,

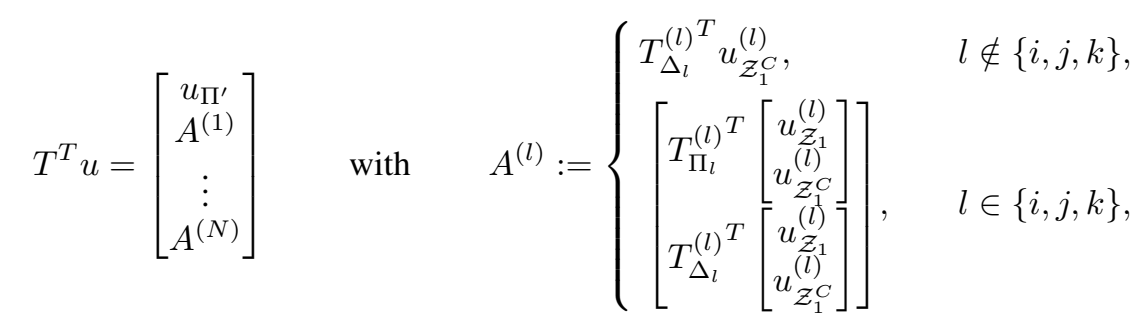

since $\Delta_{l}^{\prime}=\Delta_{l}$ for $l \notin\{i, j, k\}$, and thus we also have

$$
R R_{\mu}^{T} T^{T} u=\left[\begin{array}{c}
u_{\Pi^{\prime}} \\
\widehat{A}^{(1)} \\
\vdots \\
\widehat{A}^{(N)}
\end{array}\right]
$$

where

$$
\widehat{A}^{(l)}:=\left\{\begin{array}{cc}
A^{(l)}, & l \notin\{i, j, k\}, \\
{\left[\begin{array}{c}
\frac{1}{3}\left(T_{\Pi_{i}}^{(i) T}\left[\begin{array}{c}
u_{\mathcal{Z}_{1}}^{(i)} \\
u_{\mathcal{Z}_{1}^{C}}^{(i)}
\end{array}\right]+T_{\Pi_{j}}^{(j)^{T}}\left[\begin{array}{c}
u_{\mathcal{Z}_{1}}^{(j)} \\
u_{\mathcal{Z}^{C}}^{(j)}
\end{array}\right]+T_{\Pi_{k}}^{(k)^{T}}\left[\begin{array}{c}
u_{\mathcal{Z}_{1}}^{(k)} \\
u_{\mathcal{Z}_{1}^{C}}^{(k)}
\end{array}\right]\right) \\
T_{\Delta_{l}}^{(l)}\left[\begin{array}{c}
u_{\mathcal{Z}_{1}}^{(l)} \\
u_{\mathcal{Z}_{1}^{C}}^{(l)}
\end{array}\right]
\end{array}\right], \quad l \in\{i, j, k\} .}
\end{array}\right.
$$


Here, we have used (10.2), (10.8), and (5.1).

From (10.2) in compact form, we have

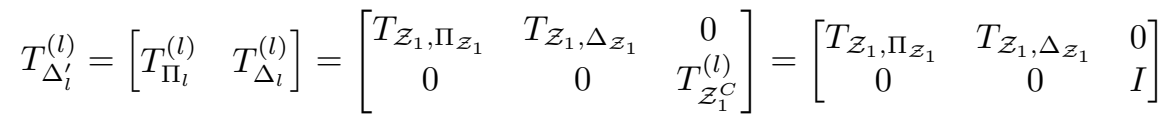

for $l \in\{i, j, k\}$ and $T_{\Delta_{l}^{\prime}}^{(l)}=T_{\Delta_{l}}^{(l)}=I$ otherwise.

We now apply $T$ to $R R_{\mu}^{T} T^{T} u$ or locally, $T_{\Delta_{l}^{\prime}}^{(l)}$ to $\widehat{A}^{(l)}$. We restrict ourselves to the case of $l \in\{i, j, k\}$ since there is nothing to show for $l \notin\{i, j, k\}$, i.e., $T_{\Delta_{l}^{\prime}}^{(l)} \widehat{A}^{(l)}=u_{\mathcal{Z}_{1}^{C}}^{(l)}=u^{(l)}$. Then, for $l \in\{i, j, k\}$, we obtain

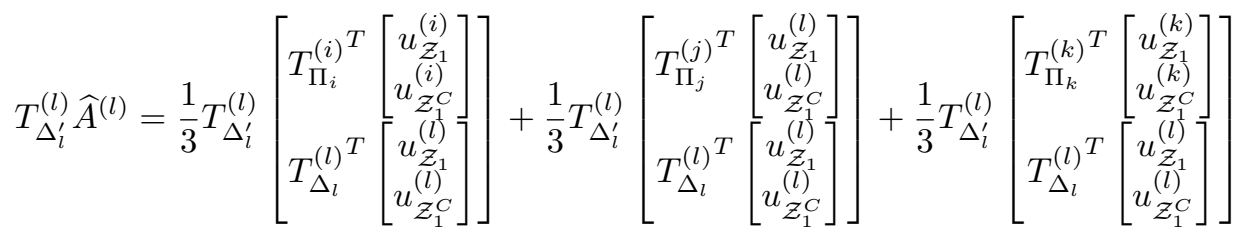

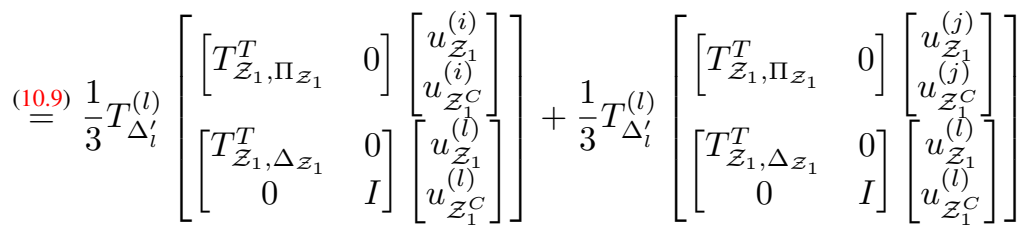

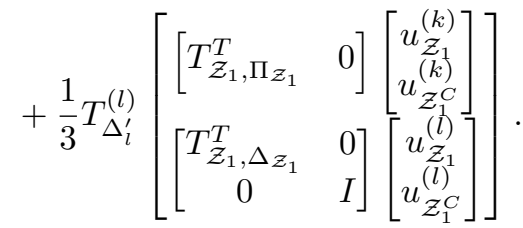

Without loss of generality, we consider $l=i$. Then, the last equation becomes

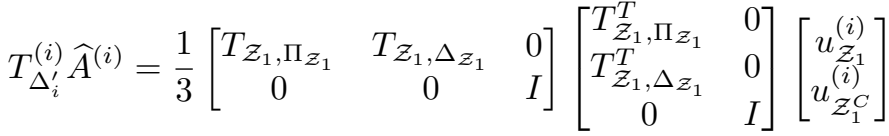

$$
\begin{aligned}
& +\frac{1}{3}\left[\begin{array}{ccc}
T_{\mathcal{Z}_{1}, \Pi} & T_{\mathcal{Z}_{1}, \Delta \mathcal{Z}_{1}} & 0 \\
0 & 0 & I
\end{array}\right]\left[\begin{array}{cccc}
T_{\mathcal{Z}_{1}, \Pi_{\mathcal{Z}_{1}}}^{T} & 0 & 0 & 0 \\
0 & 0 & T_{\mathcal{Z}_{1}, \Delta \mathcal{Z}_{1}}^{T} & 0 \\
0 & 0 & 0 & I
\end{array}\right]\left[\begin{array}{c}
u_{\mathcal{Z}_{1}}^{(j)} \\
u_{\mathcal{Z}_{1}^{C}}^{(j)} \\
u_{\mathcal{Z}_{1}}^{(i)} \\
u_{\mathcal{Z}_{1}^{C}}^{(i)}
\end{array}\right] \\
& +\frac{1}{3}\left[\begin{array}{ccc}
T_{\mathcal{Z}_{1}, \Pi \overline{\mathcal{Z}}_{1}} & T_{\mathcal{Z}_{1}, \Delta \mathcal{Z}_{1}} & 0 \\
0 & 0 & I
\end{array}\right]\left[\begin{array}{cccc}
T_{\mathcal{Z}_{1}, \Pi \mathcal{Z}_{1}}^{T} & 0 & 0 & 0 \\
0 & 0 & T_{\mathcal{Z}_{1}, \Delta}^{T} & 0 \\
0 & 0 & 0 & I
\end{array}\right]\left[\begin{array}{c}
u_{\mathcal{Z}_{1}}^{(k)} \\
u_{\mathcal{Z}_{1}^{C}}^{(k)} \\
u_{\mathcal{Z}_{1}}^{(i)} \\
u_{\mathcal{Z}_{1}^{C}}^{(i)}
\end{array}\right]
\end{aligned}
$$




$$
\begin{aligned}
& =\frac{1}{3}\left[\begin{array}{c}
u_{\mathcal{Z}_{1}}^{(i)} \\
u_{\mathcal{Z}_{1}^{C}}^{(i)}
\end{array}\right]+\frac{1}{3}\left[\begin{array}{c}
T_{\mathcal{Z}_{1}, \Pi_{\mathcal{Z}_{1}}} T_{\mathcal{Z}_{1}, \Pi_{\mathcal{Z}_{1}}}^{T} u_{\mathcal{Z}_{1}}^{(j)}+T_{\mathcal{Z}_{1}, \Delta_{\mathcal{Z}_{1}}} T_{\mathcal{Z}_{1}, \Delta_{\mathcal{Z}_{1}}}^{T} u_{\mathcal{Z}_{1}}^{(i)} \\
u_{\mathcal{Z}_{1}^{C}}^{(i)}
\end{array}\right] \\
& +\frac{1}{3}\left[\begin{array}{c}
T_{\mathcal{Z}_{1}, \Pi_{\mathcal{Z}_{1}}} T_{\mathcal{Z}_{1}, \Pi_{\mathcal{Z}_{1}}}^{T} u_{\mathcal{Z}_{1}}^{(k)}+T_{\mathcal{Z}_{1}, \Delta \mathcal{Z}_{1}} T_{\mathcal{Z}_{1}, \Delta_{\mathcal{Z}_{1}}}^{T} u_{\mathcal{Z}_{1}}^{(i)} \\
u_{\mathcal{Z}_{1}^{C}}^{(i)}
\end{array}\right] \\
& =\left[\begin{array}{c}
\frac{1}{3}\left(\left(I+2 T_{\mathcal{Z}_{1}, \Delta \mathcal{Z}_{1}} T_{\mathcal{Z}_{1}, \Delta}^{T} \Delta_{\mathcal{Z}_{1}}\right) u_{\mathcal{Z}_{1}}^{(i)}+\sum_{n \in\{j, k\}} T_{\mathcal{Z}_{1}, \Pi_{\mathcal{Z}_{1}}} T_{\mathcal{Z}_{1}, \Pi_{\mathcal{Z}_{1}}}^{T} u_{\mathcal{Z}_{1}}^{(n)}\right) \\
u_{\mathcal{Z}_{1}^{C}}^{(i)}
\end{array}\right] .
\end{aligned}
$$

This shows that we can focus on the degrees of freedom on the edge $\mathcal{Z}_{1}$ since $T R R_{\mu}^{T} T^{T}$ reduces to the identity on the degrees of freedom on $\Gamma \backslash \mathcal{Z}_{1}$, i.e., $u^{T} \widetilde{S} u=u^{T} T R_{\mu} \widehat{\widetilde{S}} R_{\mu}^{T} T^{T} u$ for $u$ with $u_{\left.\right|_{\mathcal{Z}_{1}}}=0$.

By a short computation, we obtain

$$
\begin{aligned}
& \left(I+2 T_{\mathcal{Z}_{1}, \Delta \mathcal{Z}_{1}} T_{\mathcal{Z}_{1}, \Delta_{\mathcal{Z}_{1}}}^{T}\right) u_{\mathcal{Z}_{1}}^{(i)}+\sum_{n \in\{j, k\}} T_{\mathcal{Z}_{1}, \Pi_{\mathcal{Z}_{1}}} T_{\mathcal{Z}_{1}, \Pi_{\mathcal{Z}_{1}}}^{T} u_{\mathcal{Z}_{1}}^{(n)} \\
& =\left(I+2 T_{\mathcal{Z}_{1}, \Delta_{\mathcal{Z}_{1}}} T_{\mathcal{Z}_{1}, \Delta_{\mathcal{Z}_{1}}}^{T}+2 T_{\mathcal{Z}_{1}, \Pi_{\mathcal{Z}_{1}}} T_{\mathcal{Z}_{1}, \Pi_{\mathcal{Z}_{1}}}^{T}\right) u_{\mathcal{Z}_{1}}^{(i)} \\
& -2 T_{\mathcal{Z}_{1}, \Pi_{\mathcal{Z}_{1}}} T_{\mathcal{Z}_{1}, \Pi_{\mathcal{Z}_{1}}}^{T} u_{\mathcal{Z}_{1}}^{(i)}+\sum_{n \in\{j, k\}} T_{\mathcal{Z}_{1}, \Pi_{\mathcal{Z}_{1}}} T_{\mathcal{Z}_{1}, \Pi_{\mathcal{Z}_{1}}}^{T} u_{\mathcal{Z}_{1}}^{(n)} \\
& =3 u_{\mathcal{Z}_{1}}^{(i)}+T_{\mathcal{Z}_{1}, \Pi_{\mathcal{Z}_{1}}} T_{\mathcal{Z}_{1}, \Pi_{\mathcal{Z}_{1}}}^{T}\left(u_{\mathcal{Z}_{1}}^{(j)}+u_{\mathcal{Z}_{1}}^{(k)}-2 u_{\mathcal{Z}_{1}}^{(i)}\right) .
\end{aligned}
$$

Thus, (10.10) reduces to

$$
T_{\Delta_{l}^{\prime}}^{(l)} \widehat{A}^{(l)}=\left[\begin{array}{c}
u_{\mathcal{Z}_{1}}^{(i)}+\frac{1}{3} T_{\mathcal{Z}_{1}, \Pi_{\mathcal{Z}_{1}}} T_{\mathcal{Z}_{1}, \Pi_{\mathcal{Z}_{1}}}^{T}\left(u_{\mathcal{Z}_{1}}^{(j)}+u_{\mathcal{Z}_{1}}^{(k)}-2 u_{\mathcal{Z}_{1}}^{(i)}\right) \\
u_{\mathcal{Z}_{1}^{C}}^{(i)}
\end{array}\right] .
$$

In the two following parts of the proof, we have to distinguish between $u=w_{0}$ and $u=B_{D}^{T} B w_{0}$.

First, for $u=w_{0}$ with $w_{0, \mathcal{Z}_{1}}^{(l)}:=w_{\left.0\right|_{\partial \Omega_{l} \cap \mathcal{Z}_{1}}}$, for $l \in\{i, j, k\}$, we have

$$
\begin{aligned}
u_{\mathcal{Z}_{1}}^{(j)}+u_{\mathcal{Z}_{1}}^{(k)}-2 u_{\mathcal{Z}_{1}}^{(i)} & =w_{0, \mathcal{Z}_{1}}^{(j)}+w_{0, \mathcal{Z}_{1}}^{(k)}-2 w_{0, \mathcal{Z}_{1}}^{(i)} \\
& =\left(w_{0, \mathcal{Z}_{1}}^{(j)}-w_{0, \mathcal{Z}_{1}}^{(i)}\right)+\left(w_{0, \mathcal{Z}_{1}}^{(k)}-w_{0, \mathcal{Z}_{1}}^{(i)}\right) .
\end{aligned}
$$

Since $w_{0} \in \widetilde{W}_{\widehat{Q}}$, we know from (10.5) and (10.7) that the jump across $\mathcal{Z}_{1}$ of $\left(w_{0}^{\left(r_{1}\right)}, w_{0}^{\left(r_{2}\right)}\right)$ $\left(r_{1}, r_{2} \in\{i, j, k\}, r_{1} \neq r_{2}\right)$ is orthogonal to the constraint vectors introduced before. Hence,

$$
\begin{aligned}
& T_{\mathcal{Z}_{1}, \Pi_{\mathcal{Z}_{1}}} T_{\mathcal{Z}_{1}, \Pi_{\mathcal{Z}_{1}}}^{T}\left(u_{\mathcal{Z}_{1}}^{(j)}+u_{\mathcal{Z}_{1}}^{(k)}-2 u_{\mathcal{Z}_{1}}^{(i)}\right) \\
& =T_{\mathcal{Z}_{1}, \Pi_{\mathcal{Z}_{1}}} T_{\mathcal{Z}_{1}, \Pi_{\mathcal{Z}_{1}}}^{T}\left(\left(w_{0, \mathcal{Z}_{1}}^{(j)}-w_{0, \mathcal{Z}_{1}}^{(i)}\right)+\left(w_{0, \mathcal{Z}_{1}}^{(k)}-w_{0, \mathcal{Z}_{1}}^{(i)}\right)\right)
\end{aligned}
$$

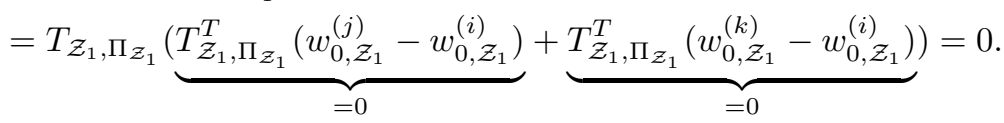

Second, consider $u=B_{D}^{T} B w_{0}$. Using $u_{\mathcal{Z}_{1}}^{(i)}=\left(B_{D}^{T} B w_{0}\right)_{\mid \partial \Omega_{i} \cap \mathcal{Z}_{1}}$, we have

$$
u_{\mathcal{Z}_{1}}^{(i)}=D_{u, \mathcal{Z}_{1}}^{(j)}\left(w_{0, \mathcal{Z}_{1}}^{(i)}-w_{0, \mathcal{Z}_{1}}^{(j)}\right)+D_{u, \mathcal{Z}_{1}}^{(k)}\left(w_{0, \mathcal{Z}_{1}}^{(i)}-w_{0, \mathcal{Z}_{1}}^{(k)}\right) .
$$


Using the corresponding formulas for $u_{\mathcal{Z}_{1}}^{(j)}$ and $u_{\mathcal{Z}_{1}}^{(k)}$, we obtain

$$
\begin{aligned}
& u_{\mathcal{Z}_{1}}^{(j)}+u_{\mathcal{Z}_{1}}^{(k)}-2 u_{\mathcal{Z}_{1}}^{(i)}=D_{u, \mathcal{Z}_{1}}^{(i)}\left(w_{0, \mathcal{Z}_{1}}^{(j)}-w_{0, \mathcal{Z}_{1}}^{(i)}\right)+D_{u, \mathcal{Z}_{1}}^{(k)}\left(w_{0, \mathcal{Z}_{1}}^{(j)}-w_{0, \mathcal{Z}_{1}}^{(k)}\right) \\
& +D_{u, \mathcal{Z}_{1}}^{(i)}\left(w_{0, \mathcal{Z}_{1}}^{(k)}-w_{0, \mathcal{Z}_{1}}^{(i)}\right)+D_{u, \mathcal{Z}_{1}}^{(j)}\left(w_{0, \mathcal{Z}_{1}}^{(k)}-w_{0, \mathcal{Z}_{1}}^{(j)}\right) \\
& \left.+2 D_{u, \mathcal{Z}_{1}}^{(j)}\left(w_{0, \mathcal{Z}_{1}}^{(j)}-w_{0, \mathcal{Z}_{1}}^{(i)}\right)+2 D_{u, \mathcal{Z}_{1}}^{(k)}\left(w_{0, \mathcal{Z}_{1}}^{(k)}-w_{0, \mathcal{Z}_{1}}^{(i)}\right)\right) \\
& =\left(D_{u, \mathcal{Z}_{1}}^{(i)}+D_{u, \mathcal{Z}_{1}}^{(j)}\right)\left(w_{0, \mathcal{Z}_{1}}^{(j)}-w_{0, \mathcal{Z}_{1}}^{(i)}\right) \\
& +\left(D_{u, \mathcal{Z}_{1}}^{(i)}+D_{u, \mathcal{Z}_{1}}^{(k)}\right)\left(w_{0, \mathcal{Z}_{1}}^{(k)}-w_{0, \mathcal{Z}_{1}}^{(i)}\right) \\
& +D_{u, \mathcal{Z}_{1}}^{(k)}\left(w_{0, \mathcal{Z}_{1}}^{(j)}-w_{0, \mathcal{Z}_{1}}^{(k)}\right)+D_{u, \mathcal{Z}_{1}}^{(k)}\left(w_{0, \mathcal{Z}_{1}}^{(k)}-w_{0, \mathcal{Z}_{1}}^{(i)}\right) \\
& \left.+D_{u, \mathcal{Z}_{1}}^{(j)}\left(w_{0, \mathcal{Z}_{1}}^{(k)}-w_{0, \mathcal{Z}_{1}}^{(j)}\right)+D_{u, \mathcal{Z}_{1}}^{(j)}\left(w_{0, \mathcal{Z}_{1}}^{(j)}-w_{0, \mathcal{Z}_{1}}^{(i)}\right)\right) \\
& =(\underbrace{D_{u, \mathcal{Z}_{1}}^{(i)}+D_{u, \mathcal{Z}_{1}}^{(j)}+D_{u, \mathcal{Z}_{1}}^{(k)}}_{=I})\left(w_{0, \mathcal{Z}_{1}}^{(j)}-w_{0, \mathcal{Z}_{1}}^{(i)}\right) \\
& +(\underbrace{D_{u, \mathcal{Z}_{1}}^{(i)}+D_{u, \mathcal{Z}_{1}}^{(j)}+D_{u, \mathcal{Z}_{1}}^{(k)}}_{=I})\left(w_{0, \mathcal{Z}_{1}}^{(k)}-w_{0, \mathcal{Z}_{1}}^{(i)}\right) \\
& =\left(w_{0, \mathcal{Z}_{1}}^{(j)}-w_{0, \mathcal{Z}_{1}}^{(i)}\right)+\left(w_{0, \mathcal{Z}_{1}}^{(k)}-w_{0, \mathcal{Z}_{1}}^{(i)}\right) .
\end{aligned}
$$

As before, the orthogonality to the constraint vectors of the jump across $\mathcal{Z}_{1}$ of $\left(w_{0}^{\left(r_{1}\right)}, w_{0}^{\left(r_{2}\right)}\right)$ $\left(r_{1}, r_{2} \in\{i, j, k\}, r_{1} \neq r_{2}\right)$ implies with (10.13)

$$
T_{\mathcal{Z}_{1}, \Pi_{\mathcal{Z}_{1}}} T_{\mathcal{Z}_{1}, \Pi_{\mathcal{Z}_{1}}}^{T}\left(u_{\mathcal{Z}_{1}}^{(j)}+u_{\mathcal{Z}_{1}}^{(k)}-2 u_{\mathcal{Z}_{1}}^{(i)}\right)=0
$$

see (10.12).

Therefore, for $u=w_{0}$ and $u=B_{D}^{T} B w_{0}$, from (10.11) with (10.12) and (10.14), we likewise have

$$
T_{\Delta_{l}^{\prime}}^{(l)} \widehat{A}^{(l)}=\left[\begin{array}{c}
u_{\mathcal{Z}_{1}}^{(l)} \\
u_{\mathcal{Z}_{1}^{C}}^{(l)}
\end{array}\right],
$$

which finally yields

$$
T R R_{\mu}^{T} T^{T} u=u
$$

for any $w_{0} \in \widetilde{W}_{\widehat{Q}}$ and $u=w_{0}$ or $u=B_{D}^{T} B w_{0}$.

Proof of Lemma 6.4. As in the proof of Lemma 6.3, we assume that a posteriori constraints are only associated with the edge $\mathcal{Z}_{1}$. 
Let $\widehat{w} \in \widetilde{W}_{T, a}$ be given. Let

$$
w_{0}:=T R \widehat{w}=T R R_{\mu}^{T} T^{T} w=T\left[\begin{array}{c}
{ }^{3}\left(\bar{w}_{\Pi_{i}}^{(i)}+\bar{w}_{\Pi_{j}}^{(j)}+\bar{w}_{\Pi_{k}}^{(k)}\right) \\
\bar{w}_{\Delta_{i}}^{(i)} \\
* \\
\frac{1}{3}\left(\bar{w}_{\Pi_{i}}^{(i)}+\bar{w}_{\Pi_{j}}^{(j)}+\bar{w}_{\Pi_{k}}^{(k)}\right) \\
\bar{w}_{\Delta_{j}}^{(j)} \\
* \\
\frac{1}{3}\left(\bar{w}_{\Pi_{i}}^{(i)}+\bar{w}_{\Pi_{j}}^{(j)}+\bar{w}_{\Pi_{k}}^{(k)}\right) \\
\bar{w}_{\Delta_{k}}^{k(k)} \\
*
\end{array}\right] .
$$

By construction, we have $\widehat{B} \widehat{w}=B T R \widehat{w}=B w_{0}$. Then, with $\widehat{w}_{0, \Pi}:=\frac{1}{3}\left(\bar{w}_{\Pi_{i}}^{(i)}+\bar{w}_{\Pi_{j}}^{(j)}+\bar{w}_{\Pi_{k}}^{(k)}\right)$ and

$$
w_{0, \Delta_{l}^{\prime}}^{(l)}:=\left[\begin{array}{ll}
T_{\Pi_{l}}^{(l)} & T_{\Delta_{l}}^{(l)}
\end{array}\right]\left[\begin{array}{c}
\widehat{w}_{0, \Pi} \\
\bar{w}_{\Delta_{l}}^{(l)}
\end{array}\right], \quad l \in\{i, j, k\}
$$

we also have

$$
\widehat{w}_{0, \Pi}=T_{\Pi_{r_{1}}}^{\left(r_{1}\right) T} w_{0, \Delta_{r_{1}}^{\prime}}^{\left(r_{1}\right)}=T_{\Pi_{r_{2}}}^{\left(r_{2}\right) T} w_{0, \Delta_{r_{2}}^{\prime}}^{\left(r_{2}\right)}
$$

for $r_{1}, r_{2} \in\{i, j, k\}, r_{1} \neq r_{2}$. From the construction of $T_{\Pi_{l}}^{(l)}, l \in\{i, j, k\}$, it follows that the jump across $\mathcal{Z}_{1}$ of $\left(w_{0}^{\left(r_{1}\right)}, w_{0}^{\left(r_{2}\right)}\right)$ is orthogonal to the constraint vectors; cf. (10.1), (10.5), and (10.7). Since the constraints are local and we have assumed that a posteriori constraints are only associated with the edge common to $\Omega_{i}, \Omega_{j}$, and $\Omega_{k}$, we find also that all other local combinations $\left(w_{0}^{\left(r_{1}\right)}, w_{0}^{\left(r_{2}\right)}\right), r_{1} \neq r_{2}, r_{1}, r_{2} \in\{1, \ldots, N\}$, satisfy the constraints. Thus, $w_{0}$ fulfills all constraints introduced before, i.e., $w_{0} \in \widetilde{W}_{\widehat{Q}}$. More general cases can be treated analogously.

Let $w_{0} \in \widetilde{W}_{\widehat{Q}}$ be given. By the first identity of Lemma 6.3, we have $w_{0}=T R R_{\mu}^{T} T^{T} w_{0}$. Define $\widehat{w}:=R_{\mu}^{T} T^{T} w_{0}$. It yields

$$
B w_{0}=B T R R_{\mu}^{T} T^{T} w_{0}=\widehat{B} \widehat{w} .
$$

Again, more general cases can be treated analogously.

Proof of Lemma 6.5. Let $\widehat{w} \in \widetilde{W}_{T, a}$ be given. Then, by using the first part of Lemma 6.4 and the second part of Lemma 6.3, we have

$$
\begin{aligned}
\left|\widehat{P}_{D} \widehat{w}\right|^{2} & =\widehat{\widetilde{w}}^{T} \widehat{B}^{T} \widehat{B}_{D} \widehat{\widetilde{S}} \widehat{B}_{D}^{T} \widehat{B} \widehat{w}=w_{0} B^{T} \widehat{B}_{D} \widehat{\widetilde{S}} \widehat{B}_{D}^{T} B w_{0} \\
& =w_{0} B^{T} B_{D} \widetilde{S} B_{D}^{T} B w_{0}=\left|P_{D} w_{0}\right|_{\widetilde{S}}^{2}
\end{aligned}
$$

with $w_{0}:=T R \widehat{w} \in \widetilde{W}_{\widehat{Q}}$.

Let $w_{0} \in \widetilde{W}_{\widehat{Q}}$ be given. Then, by using the second part of Lemma 6.3 and the second part of Lemma 6.4, we have

$$
\left|P_{D} w_{0}\right|_{\widetilde{S}}^{2}=w_{0}^{T} P_{D}^{T} \widetilde{S} P_{D} w_{0}=\widehat{w}^{T} \widehat{B}^{T} \widehat{B}_{D} \widehat{\widetilde{S}} \widehat{B}_{D}^{T} \widehat{B} \widehat{w}=\left|\widehat{P}_{D} \widehat{w}\right|_{\widetilde{\widetilde{S}}}^{2}
$$


with $\widehat{w}:=R_{\mu}^{T} T^{T} w_{0} \in \widetilde{W}_{T, a}$.

Proof of Lemma 6.6. By the arguments from Lemma 6.4, Lemma 6.3, and the identity $B P_{D} w=B w$ for $w \in \widetilde{W}$ from [43, equation (8.1)] and $\widetilde{W} \widehat{Q} \subset \widetilde{W}$, with $w_{0}:=T R \widehat{w}$, we have,

$\widehat{B} \widehat{P}_{D} \widehat{w}=\widehat{B} \widehat{B}_{D}^{T} \widehat{B} \widehat{w}=\widehat{B} \widehat{B}_{D}^{T} B w_{0}=B T R R_{\mu}^{T} T^{T} B_{D}^{T} B w_{0}=B B_{D}^{T} B w_{0}=B w_{0}=\widehat{B} \widehat{w}$.

\section{REFERENCES}

[1] L. Beirão da Veiga, L. F. PAVARino, S. SCACChi, O. B. Widlund, and S. ZamPini, Adaptive selection of primal constraints for isogeometric BDDC deluxe preconditioners, SIAM J. Sci. Comput., 39 (2017), pp. A281-A302.

[2] - Isogeometric BDDC preconditioners with deluxe scaling, SIAM J. Sci. Comput., 36 (2014), pp. A1118A1139.

[3] P. BJøRSTAD, J. Koster, AND P. KRZYZANOwSKi, Domain decomposition solvers for large scale industrial finite element problems, in Applied Parallel Computing. New Paradigms for HPC in Industry and Academia, T. Sørevik, F. Manne, A. H. Gebremedhin, and R. Moe, eds., vol. 1947 of Lect. Notes Comput. Sci., Springer, Berlin, 2001, pp. 373-383.

[4] P. BJøRSTAD AND P. KRZYZANOWSKI, A flexible 2-level Neumann-Neumann method for structural analysis problems, in Parallel Processing and Applied Mathematics, R. Wyrzykowski, J. Dongarra, M. Paprzycki, and Jerzy Waśniewski, eds., vol. 2328 of Lect. Notes Comput. Sci., Springer, Berlin, 2002, pp. 387-394.

[5] C. Bovet, A. Parret-Fréaud, N. Spillane, and P. Gosselet, Adaptive multipreconditioned FETI: scalability results and robustness assessment, Computers \& Structures, 193 (2017), pp. 1-20.

[6] J. G. CALVO, A BDDC algorithm with deluxe scaling for $H$ (curl) in two dimensions with irregular subdomains, Math. Comp., 85 (2016), pp. 1085-1111.

[7] J. G. CALVO AND O. B. WIDLUND, An adaptive choice of primal constraints for BDDC domain decomposition algorithms, Electron. Trans. Numer. Anal., 45 (2016), pp. 524-544.

http://etna.ricam.oeaw.ac.at/vol.45.2016/pp524-544.dir/pp524-544.pdf

[8] E. T. CHUNG AND H. H. KIM, A deluxe FETI-DP algorithm for a hybrid staggered discontinuous Galerkin method for H(curl)-elliptic problems, Internat. J. Numer. Meth. Engng., 98 (2014), pp. 1-23.

[9] J. M. CROS, A preconditioner for the Schur complement domain decomposition method, in Domain Decomposition Methods in Science and Engineering, I. Herrera, D. E. Keyes, O. B. Widlund, R. Yates, eds., National Autonomous University of Mexico (UNAM), Mexico City, 2003, pp. 373-380.

[10] C. R. DOHRMANn, A preconditioner for substructuring based on constrained energy minimization, SIAM J. Sci. Comput., 25 (2003), pp. 246-258.

[11] - An approximate BDDC preconditioner, Numer. Linear Algebra Appl., 14 (2007), pp. 149-168.

[12] C. Dohrmann AND C. PeChstein, Modern domain decomposition solvers - BDDC, deluxe scaling, and an algebraic approach, Talk by C. Pechstein at the University Linz, December 2013. http://people.ricam.oeaw.ac.at/c.pechstein/pechstein-bddc2013.pdf

[13] C. R. DOHRMANn AND O. B. WidLund, Some recent tools and a BDDC algorithm for 3D problems in H(curl), in Domain Decomposition Methods in Science and Engineering XX, R. Bank, M. Holst, O. Widlund, and J. Xu, eds., vol. 91 of Lect. Notes Comput. Sci. Eng., Springer, Heidelberg, 2013, pp. $15-25$.

[14] V. Dolean, F. NataF, R. Scheichl, AND N. SpIllane, Analysis of a two-level Schwarz method with coarse spaces based on local Dirichlet-to-Neumann maps, Comput. Methods Appl. Math., 12 (2012), pp. 391-414.

[15] Z. DostáL, Conjugate gradient method with preconditioning by projector, Int. J. Comput. Math., 23 (1988), pp. 315-323.

[16] - Projector preconditioning and domain decomposition methods, Appl. Math. Comput, 37 (1990), pp. $75-81$.

[17] E. Eikeland, L. MARCINKOWSKI, AND T. RAhMAN, Overlapping Schwarz methods with adaptive coarse spaces for multiscale problems in $3 D$, Preprint on arXiv, 2016. https://arxiv.org/abs/1611.00968

[18] C. Farhat, M. Lesoinne, P. LeTallec, K. Pierson, and D. RiXen, FETI-DP: a dual-primal unified FETI method. I. A faster alternative to the two-level FETI method, Internat. J. Numer. Methods Engrg., 50 (2001), pp. 1523-1544.

[19] C. Farhat, M. Lesoinne, AND K. Pierson, A scalable dual-primal domain decomposition method, Numer. Linear Algebra Appl., 7 (2000), pp. 687-714. 
[20] Y. FRAGAKIS AND M. PAPADRAKAKIS, The mosaic of high performance domain decomposition methods for structural methanics: formulation, interrelation and numerical efficiency of primal and dual methods, Comput. Methods Appl. Mech. Engrg., 192 (2003), pp. 3799-3830.

[21] J. GALVIS AND Y. EFENDIEv, Domain decomposition preconditioners for multiscale flows in high-contrast media, Multiscale Model. Simul., 8 (2010), pp. 1461-1483.

[22] - Domain decomposition preconditioners for multiscale flows in high contrast media: reduced dimension coarse spaces, Multiscale Model. Simul., 8 (2010), pp. 1621-1644.

[23] M. J. GANDER, A. LONELAND, AND T. RAHMAN, Analysis of a new harmonically enriched multiscale coarse space for domain decomposition methods, Preprint on arXiv, 2015. https://arxiv.org/abs/1512.05285

[24] S. Gippert, A. Klawonn, AND O. Rheinbach, A deflation based coarse space in Dual-Primal FETI methods for almost incompressible elasticity, in Numerical Mathematics and Advanced Applications ENUMATH 2013, A. Abdulle, S. Deparis, D. Kressner, F. Nobile, and M. Picasso, eds., vol. 103 of Lect. Notes Comput. Sci. Eng., Springer, Cham, 2015, pp. 573-581.

[25] P. Goldfeld, L. F. PaVARino, And O. B. Widlund, Balancing Neumann-Neumann preconditioners for mixed approximations of heterogeneous problems in linear elasticity, Numer. Math., 95 (2003), pp. 283-324.

[26] A. Heinlein, A. Klawonn, J. Knepper, And O. Rheinbach, Multiscale coarse spaces for overlapping Schwarz methods based on the ACMS space in $2 d$, Tech. Report 09/2016, Fakultät für Mathematik und Informatik, TU Bergakademie Freiberg, Freiberg, 2016.

http://tu-freiberg.de/fakult1/forschung/preprints

[27] M. Jarošová, A. Klawonn, AND O. Rheinbach, Projector preconditioning and transformation of basis in FETI-DP algorithms for contact problems, Math. Comput. Simulation, 82 (2012), pp. 1894-1907.

[28] G. KARYPIS AND V. KUMAR, Metis, unstructured graph partitioning and sparse matrix ordering system. version 2.0, Tech. Report, University of Minnesota, Department of Computer Science, Minneapolis, August 1995.

[29] _ - A fast and high quality multilevel scheme for partitioning irregular graphs, SIAM J. Sci. Comput., 20 (1998), pp. 359-392.

[30] H. H. KIM AND E. T. CHUNG, A BDDC algorithm with enriched coarse spaces for two-dimensional elliptic problems with oscillatory and high contrast coefficients, Multiscale Model. Simul., 13 (2015), pp. 571593.

[31] H. H. KIM, E. CHUNG, AND J. WANG, BDDC and FETI-DP preconditioners with adaptive coarse spaces for three-dimensional elliptic problems with oscillatory and high contrast coefficients, J. Comput. Phys., 349 (2017), pp. 191-214.

[32] A. KLAWONN, M. KÜHN, AND O. RHEINBACH, Adaptive coarse spaces for FETI-DP in three dimensions, SIAM J. Sci. Comput., 38 (2016), pp. A2880-A2911.

[33] - Adaptive FETI-DP and BDDC methods with a generalized transformation of basis for heterogeneous problems, Electron. Trans. Numer. Anal., 49 (2018), pp. 1-27. http://etna.ricam.oeaw.ac.at/vol.49.2018/pp1-27.dir/pp1-27.pdf

[34] _ FETI-DP and BDDC methods with a transformation of basis for heterogeneous problems: Connections to deflation, Tech. Report 01/2017, Fakultät für Mathematik und Informatik, TU Bergakademie Freiberg, Freiberg, April 2017. http://tu-freiberg.de/fakult1/forschung/preprints.

[35] — Parallel adaptive FETI-DP using lightweight asynchronous dynamic load balancing, Internat. J. Numer. Methods Engrg., published online Sept. 12, 2019, doi: 10.1002/nme.6237.

[36] A. Klawonn, M. LANSER, AND O. RHEInBACH, Toward extremely scalable nonlinear domain decomposition methods for elliptic partial differential equations, SIAM J. Sci. Comput., 37 (2015), pp. C667-C696.

[37] A. KLAWOnN, P. RADTKE, AND O. RHEInBACH, FETI-DP methods with an adaptive coarse space, SIAM J. Numer. Anal., 53 (2015), pp. 297-320.

[38] - A comparison of adaptive coarse spaces for iterative substructuring in two dimensions, Electron. Trans. Numer. Anal., 45 (2016), pp. 75-106.

http://etna.ricam.oeaw.ac.at/vol.45.2016/pp75-106.dir/pp75-106.pdf

[39] A. KLAWONN AND O. RHEInBACH, A parallel implementation of dual-primal FETI methods for threedimensional linear elasticity using a transformation of basis, SIAM J. Sci. Comput., 28 (2006), pp. 18861906.

[40] - Robust FETI-DP methods for heterogeneous three dimensional elasticity problems, Comput. Methods Appl. Mech. Engrg., 196 (2007), pp. 1400-1414.

[41] - Deflation, projector preconditioning, and balancing in iterative substructuring methods: connections and new results, SIAM J. Sci. Comput., 34 (2012), pp. A459-A484.

[42] A. KLAWONn AND O. B. WIDLUND, FETI and Neumann-Neumann iterative substructuring methods: connections and new results, Comm. Pure Appl. Math., 54 (2001), pp. 57-90.

[43] - Dual-primal FETI methods for linear elasticity, Comm. Pure Appl. Math., 59 (2006), pp. 1523-1572. 
[44] A. Klawonn, O. B. Widlund, And M. DRYja, Dual-primal FETI methods for three-dimensional elliptic problems with heterogeneous coefficients, SIAM J. Numer. Anal., 40 (2002), pp. 159-179.

[45] - Dual-primal FETI methods with face constraints, in Recent Developments in Domain Decomposition Methods, L. F. Pavarino and A. Toselli, eds., vol. 23 of Lect. Notes Comput. Sci. Eng., Springer, Berlin, 2002, pp. 27-40.

[46] M. J. KÜHN, Adaptive FETI-DP and BDDC methods for highly heterogeneous elliptic finite element problems in three dimensions, $\mathrm{PhD}$. Thesis, Mathematisch-Naturwissenschaftlichen Fakultät, Universität zu Köln, Köln, 2018.

[47] J. Li AND O. B. WidLund, FETI-DP, BDDC, and block Cholesky methods, Internat. J. Numer. Meth. Engng., 66 (2006), pp. 250-271.

[48] J. MAndel, C. R. Dohrmann, AND R. Tezaur, An algebraic theory for primal and dual substructuring methods by constraints, Appl. Numer. Math., 54 (2005), pp. 167-193.

[49] J. MANDEL AND B. SousEDíK, Adaptive selection of face coarse degrees of freedom in the BDDC and the FETI-DP iterative substructuring methods, Comput. Methods Appl. Mech. Engrg., 196 (2007), pp. 1389-1399.

[50] J. MANDEL, B. Sousedík, AND J. ŠísteK, Adaptive BDDC in three dimensions, Math. Comput. Simulation, 82 (2012), pp. 1812-1831.

[51] R. NABBEN AND C. VUIK, A comparison of deflation and the balancing preconditioner, SIAM J. Sci. Comput., 27 (2006), pp. 1742-1759.

[52] F. Nataf, H. Xiang, V. Dolean, and N. Spillane, A coarse space construction based on local Dirichletto-Neumann maps, SIAM J. Sci. Comput., 33 (2011), pp. 1623-1642.

[53] R. A. Nicolaides, Deflation of conjugate gradients with applications to boundary value problems, SIAM J. Numer. Anal., 24 (1987), pp. 355-365.

[54] D.-S. OH, O. B. Widlund, S. ZAmpini, AND C. R. Dohrmann, BDDC Algorithms with deluxe scaling and adaptive selection of primal constraints for Raviart-Thomas vector fields, Math. Comp., 87 (2018), pp. 659-692.

[55] C. PeChSTEIn AND C. R. Dohrmann, A unified framework for adaptive BDDC, Electron. Trans. Numer. Anal., 46 (2017), pp. 273-336. http://etna.ricam.oeaw.ac.at/vol.46.2017/pp273-336.dir/pp273-336.pdf

[56] C. PECHSTEIN AND R. SCHEICHL, Analysis of FETI methods for multiscale PDEs. Part II: interface variation, Numer. Math., 118 (2011), pp. 485-529.

[57] O. RHEINBACH, Parallel scalable iterative substructuring: Robust exact and inexact FETI-DP methods with applications to elasticity, PhD. Thesis, Fachbereich Mathematik, Universität Duisburg-Essen, Duisburg, 2006.

[58] _ - Parallel iterative substructuring in structural mechanics, Arch. Comput. Methods Eng., 16 (2009), pp. $425-463$.

[59] M. V. SARKIS MARTINS, Schwarz preconditioners for elliptic problems with discontinuous coefficients using conforming and non-conforming elements, PhD. Thesis, Department of Mathematics, New York University, New York, 1994.

[60] N. Spillane, V. Dolean, P. Hauret, F. Nataf, C. Pechstein, and R. Scheichl, abstract robust coarse spaces for systems of PDEs via generalized eigenproblems in the overlaps, Numer. Math., 126 (2014), pp. 741-770.

[61] N. SPILLANE AND D. RIXEN, Automatic spectral coarse spaces for robust FETI and BDD algorithms, Internat. J. Numer. Methods Engng., 95 (2013), pp. 953-990.

[62] A. Toselli And O. B. Widlund, Domain Decomposition Methods-Algorithms and Theory, Springer, Berlin, 2005.

[63] S. ZAMPINI, PCBDDC: a class of robust dual-primal methods in PETSc, SIAM J. Sci. Comput., 38 (2016), pp. S282-S306.

[64] S. ZAMPINI AND X. TU, Multilevel balancing domain decomposition by constraints deluxe algorithms with adaptive coarse spaces for flow in porous media, SIAM J. Sci. Comput., 39 (2017), pp. A1389-A1415. 